INRA Prod. Anim.,

2008, 21 (4), 325-344

\title{
Connaissance du devenir des éléments à risques dans les différentes filières de gestion des effluents porcins
}

\author{
M. BONNEAU', F. BELINE', J.-Y. DOURMAD', M. HASSOUNA ${ }^{3}$, C. JONDREVILLE I,6, L. LOYON², T. MORVAN³, \\ J.-M. PAILLAT'4, Y. RAMONET ${ }^{5}$, P. ROBIN 3 \\ ${ }^{1}$ INRA, UMR 1079 Systèmes d'Elevage, Nutrition Animale et Humaine, F-35590 Saint-Gilles, France \\ 2 Cemagref, UR Gestion environnementale et traitement biologique des déchets, F-35044 Rennes, France \\ 3 INRA, UMR 1069 Sol, Agro-hydrosystèmes, Spatialisation, F-35042 Rennes France \\ ${ }^{4}$ CIRAD, UPR Recyclage et Risque, F-35042 Rennes France \\ ${ }^{5}$ Chambre Régionale d'Agriculture de Bretagne, F-35042 Rennes France \\ ${ }^{6}$ Adresse actuelle : INRA, USC340 Animal et fonctionnalités des produits animaux, \\ F-54505 Vandoeuvre-lès-Nancy, France \\ Courriel : Michel.Bonneau@rennes.inra.fr
}

\begin{abstract}
Pour appréhender l'impact de la production porcine sur l'environnement, il faut avoir une bonne connaissance du devenir des éléments considérés comme à risque pour l'environnement. Le programme «Porcherie verte» s'est particulièrement attaché aux éléments présents dans l'alimentation des animaux dont la fraction non retenue dans la masse corporelle est éliminée dans les urines et les fèces et contribue à constituer les effluents d'élevage (cf. tableau 1 de l'introduction générale). Nous avons voulu mieux connaître les mécanismes mis en œuvre dans la transformation de ces éléments tout au long de la chaîne, depuis l'alimentation du bétail jusqu'à l'épandage sur le sol, établir dans quelle mesure on peut maîtriser le devenir de ces éléments et contrôler les quantités émises, et enfin mesurer les différences observées entre les différentes filières de gestion des effluents (cf. figure 1 de l'introduction générale).
\end{abstract}

La plupart des publications issues $\mathrm{du}$ programme «Porcherie verte» présentent les résultats de façon horizontale, en se focalisant sur une étape particulière des filières de gestion des effluents. Dans cet article, nous avons pris le parti de présenter les résultats par élément (azote, phosphore, éléments traces métalliques), de façon à rapprocher les résultats obtenus aux différents niveaux des filières de gestion des effluents. La dernière section de cet article essaie de faire la synthèse des connaissances acquises. Elle s'appuie à cet effet, sur les figures 25 à 27 qui tentent de montrer comment ce programme a contribué à faire avancer les connaissances dans le domaine. Nous renvoyons le lecteur aux autres articles de ce numéro pour la présentation des résultats relatifs aux émissions gazeuses, aux nuisances olfactives ou pour des approches plus globalisantes.

En ce qui concerne l'azote, l'objectif est de bien connaître les quantités disponibles à chaque étape de la gestion des effluents afin de pouvoir ajuster au mieux les quantités d'élément à épandre aux besoins des cultures qui exploiteront la valeur fertilisante de cet élément. La maîtrise par voie alimentaire des quantités excrétées par les animaux était déjà bien documentée avant le démarrage du programme «Porcherie verte». Nous avons voulu cependant aller au-delà en mesurant les conséquences d'un changement dans la teneur en protéines de l'aliment distribué aux animaux sur les étapes ultérieures de la gestion des lisiers, jusqu'à l'épandage sur le sol (cf. §1.1). L'abattement d'azote permis par le traitement biologique des lisiers était aussi bien documenté mais une comparaison des performances des différents procédés technologiques utilisables méritait d'être effectuée (cf. §1.4). Alors que le devenir de l'azote dans les filières «Lisier» était relativement bien connu, il n'en allait pas de même dans celles utilisant les litières ou le compostage. Il convenait d'établir avec plus de précision les pertes d'azote associées aux différents types de litières (cf. §1.2) et aux différentes techniques de compostage (cf. §1.3). Enfin, un effort méthodologique important a été consenti pour mettre au point une méthode de prédiction de la valeur fertilisante azotée des effluents porcins (cf. $§ 1.5$ ).
En ce qui concerne le phosphore, il s'agit, comme précédemment exposé pour l'azote, de bien ajuster les quantités épandues aux besoins des cultures. La majeure partie de l'effort a été ici consenti au niveau de la maîtrise des quantités excrétées par l'animal par voie alimentaire car les connaissances dans ce domaine étaient très parcellaires au moment du démarrage du programme «Porcherie verte». Pour ce faire, il faut bien connaître les besoins en phosphore des animaux pour ajuster au mieux les apports alimentaires (cf. $\$ 2.1$ ) et améliorer la digestibilité du phosphore pour limiter les excédents d'apports visant à pallier un manque de disponibilité de cet élément pour l'animal (cf. §2.2). Nous avons aussi voulu faire un bilan de l'abattement de phosphore permis par les différents procédés de séparation de phases (cf. \$2.3). Enfin, la valeur fertilisante des différents types d'effluents a été mesurée (cf. §2.4).

La gestion des éléments traces métalliques diffère de celle de l'azote et du phosphore. Les apports de $\mathrm{Cu}$ et de $\mathrm{Zn}$ au sol ne sont pas considérés pour leur valeur fertilisante car les besoins des cultures sont faibles en regard des quantités présentes dans les effluents. Ils sont pris en compte sous l'angle de la menace que leur accumulation excessive fait peser sur la fertilité des sols. Ces éléments sont en effet phytotoxiques au-delà de certaines concentrations et ils sont réputés se fixer de façon irréversible dans le sol, comme le confirment nos mesures (cf. §3.4). Par ailleurs ils sont réputés difficiles à extraire des effluents par 
traitement, même si nous avons montré que certains procédés de séparation de phases permettaient d'atteindre partiellement cet objectif (cf. \$3.3). C'est pour cette raison qu'un grand effort a été consenti pour essayer de maîtriser les rejets de ces éléments par voie alimentaire. Comme pour le phosphore, il s'agit de mieux connaître les besoins réels des animaux (cf. \$3.1) et d'améliorer leur disponibilité (cf. §3.2). Les simulations que nous avons réalisées montrent que l'incorporation de doses élevées d'éléments traces métalliques dans les aliments pour améliorer la santé et les performances des animaux est incompatible avec une préservation à long terme du capital de fertilité des sols (cf. §3.5).

\section{1 / L'azote}

Les termes du bilan de l'azote dans une exploitation avec un atelier de production porcine (figure 1) s'établissent de façon assez commode en ce qui concerne les entrées. Du côté de l'atelier porcin on comptabilise essentiellement l'azote contenu dans les aliments des animaux, auquel se rajoutent celui contenu dans la masse corporelle des animaux entrant dans l'exploitation et,

Figure 2. Représentation simplifiée des flux d'azote chez le porc en croissance (lisier sans traitement).

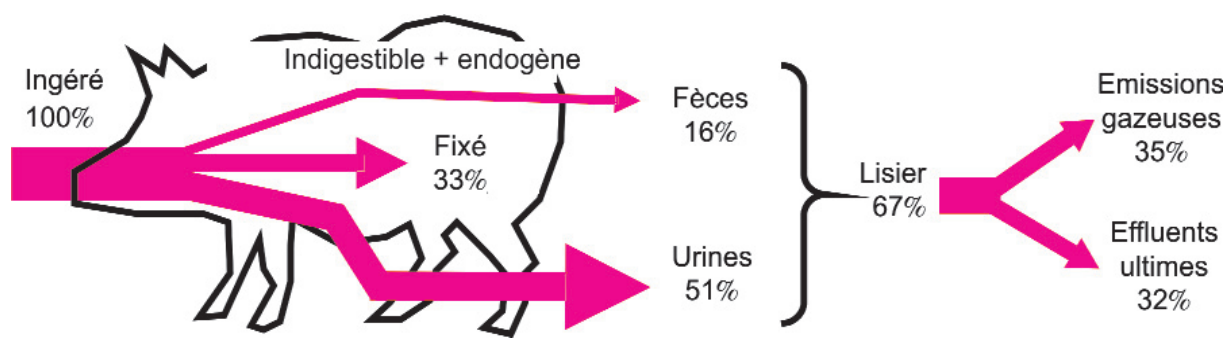

le cas échéant, celui contenu dans les litières. Au niveau global de l'exploitation, il faut y ajouter toutes les importations de fertilisants azotés, minéraux ou organiques et éventuellement de litières. Les sorties d'azote sont par contre beaucoup plus difficiles à quantifier en raison de la multiplicité des formes (solides, solubles, gazeuses) et des sites (animal, bâtiment, stockage des effluents, traitement, épandage, sol...) de sortie.

Une partie de l'azote alimentaire ingéré est retenu par l'animal sous forme de protéines corporelles (figure 2). Le reste est excrété sous forme solide dans les fèces (essentiellement sous forme de protéines, qu'il s'agisse

Figure 1. Représentation simplifiée des chemins possibles du cycle de l'azote sur le territoire d'une exploitation comprenant un atelier de production porcine. Les éléments en rouge figurent les flux d'entrée $(--\rightarrow$ : aliment, litière, fertilisants) et de sortie ( $\longrightarrow$ : produits et coproduits exportés, lessivage ; 0 : émissions gazeuses). Les flèches noires représentent les flux internes à l'exploitation.

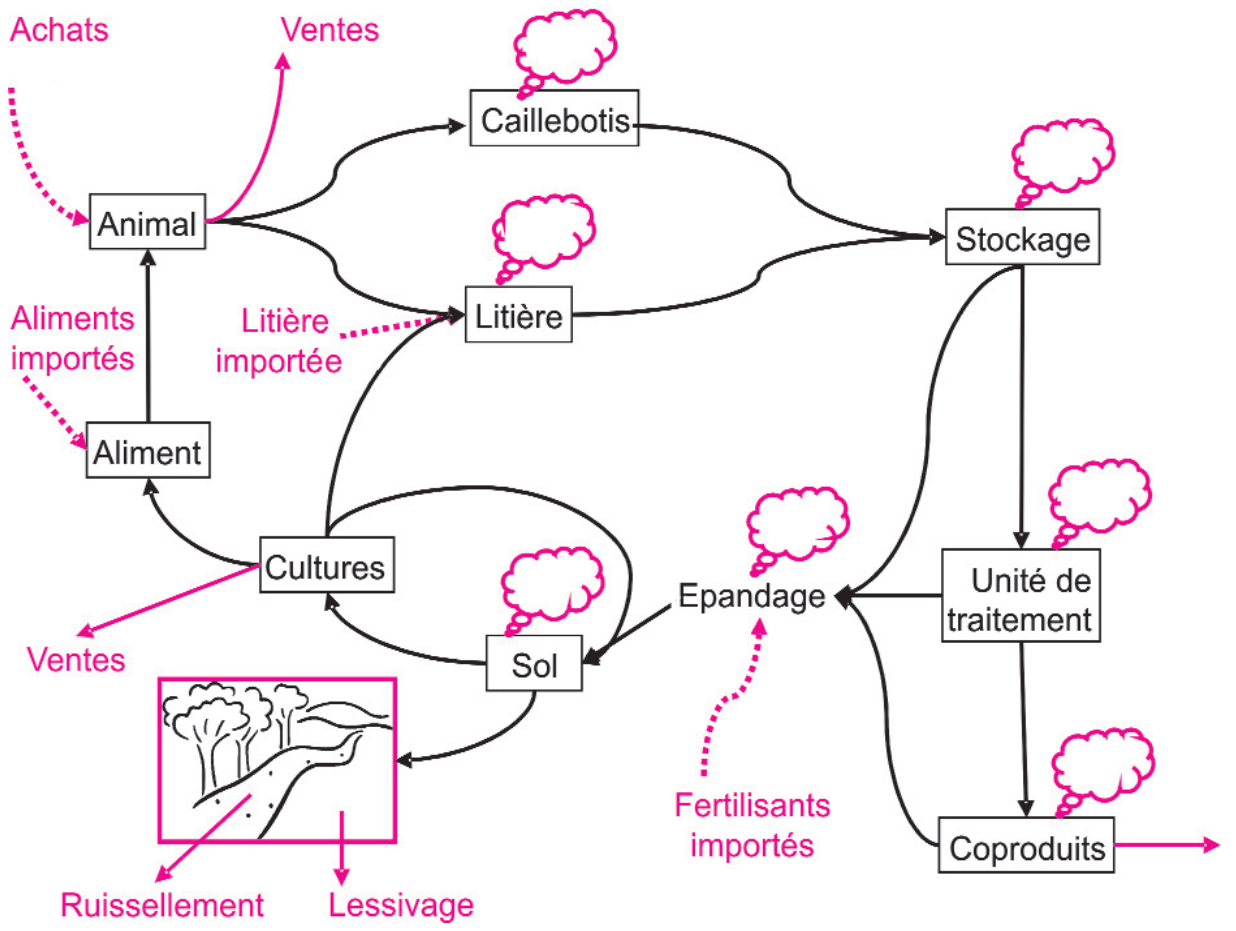
effluents animaux est déterminée par l'azote minéral, immédiatement disponible, et la minéralisation progressive de l'azote organique, qui constitue la fraction majoritaire des effluents solides. La minéralisation de l'azote suit généralement une dynamique complexe, marquée le plus souvent par des phases successives d'organisation et de minéralisation d'amplitude et de durée très variables entre produits.

Les lisiers contiennent majoritairement de l'azote soluble immédiatement disponible pour les plantes. Il faudra donc bien raisonner les périodes d'épandage pour que les apports d'azote soient adaptés aux besoins instantanés des plantes. L'azote des fumiers et composts est par contre majoritairement organisé sous des formes qui ne sont pas immédiatement assimilables par les cultures. Ce n'est qu'après avoir été minéralisé dans le sol qu'il pourra participer à la nutrition des cultures. 
Figure 3. Flux comparés d'azote (en g par animal et par jour) de l'ingestion par l'animal au sol, lors de l'apport de trois aliments différant par leur teneur en protéines totales, mais permettant les mêmes performances de croissance (d'après Portejoie et al 2004). L'azote ingéré se répartit au niveau de l'animal entre une partie fixée dans les protéines corporelles (grisé) et une partie excrétée dans les urines ou les fèces qui se mélangent pour former le lisier. Une partie de l'azote est volatilisé dans le bâtiment d'élevage (noir plein), pendant le stockage du lisier (rouge plein) et au cours de l'épandage (lignes horizontales rouges). Le reste se retrouve dans le sol (hachuré noir).

\section{$12 \%$ protéines}

$16 \%$ protéines

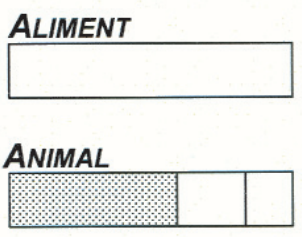

\section{BATIMENT}
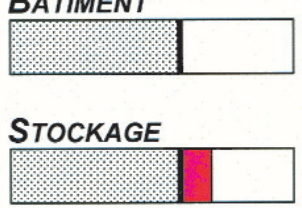

EPANDAGE

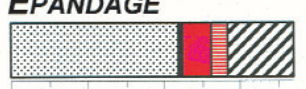

$\begin{array}{llll}0 & 10 & 20 & 30\end{array}$
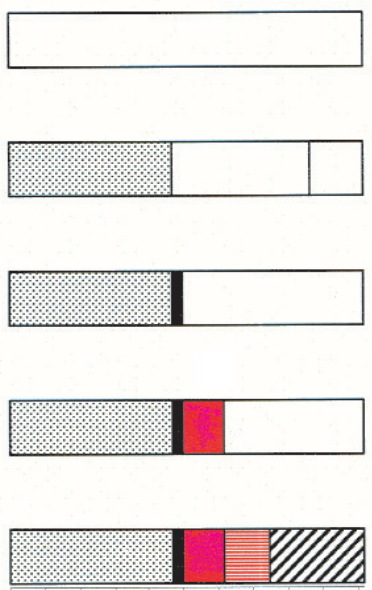

$\begin{array}{llllll}0 & 10 & 20 & 30 & 40 & 50\end{array}$
$20 \%$ protéines

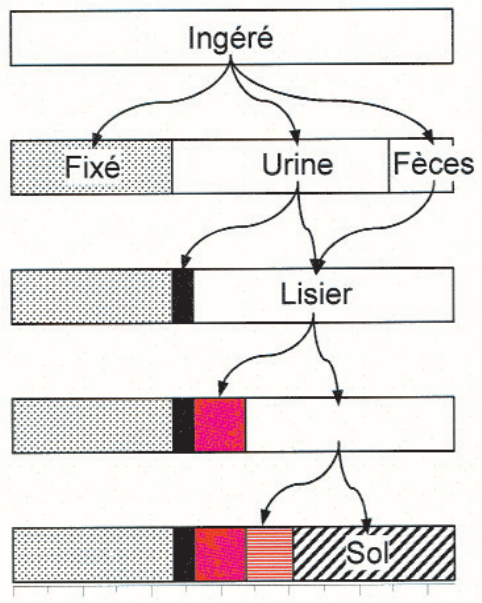

Flux d'azote (g par animal et par jour)
Gaz batiment
目 Gaz épandage

ⓞl

\section{1 / La diminution de la teneur en protéines de l'aliment permet de réduire fortement les quanti- tés d'azote excrétées par les ani- maux}

Portejoie et al (2002, 2004) ont mesuré les flux d'azote générés par des animaux en engraissement recevant trois régimes différant par leur teneur en protéines, mais apportant les mêmes quantités d'acides aminés indispensables. Les porcs étaient logés sur caillebotis et aucun traitement n'était effectué sur les lisiers.

Pour une même quantité d'azote corporel retenu $(22,23$ et $23 \mathrm{~g}$ par porc et par jour pour les régimes à 20,16 et $12 \%$ de protéines, respectivement) la réduction de la teneur en protéines de l'aliment permet de diminuer sensiblement les quantités quotidiennes d'azote arrivant jusqu'au sol $(23,14$ et $9 \mathrm{~g})$ et volatilisées dans l'atmosphère $(17,14$ et $6 \mathrm{~g}$, figure 3). La réduction de la teneur en protéines de l'aliment améliore le rendement de fixation de l'azote alimentaire en azote corporel (figure 4, partie gauche), mais ne change pas sensiblement la répartition de l'azote excrété par les animaux (figure 4, partie droite) entre l'atmosphère et le sol, où il est potentiellement valorisable par les plantes, sous réserve de ne pas être épandu en excès.

\section{2 / L'utilisation de litières conduit à une perte importante de l'azote excrété par les ani- maux}

En bâtiment d'élevage, la gestion des effluents sous forme solide conduit à des pertes d'azote plus importantes qu'avec une gestion sous forme liquide. Les fumiers sont en effet le siège de réactions de nitrification/dénitrification qui aboutissent à la volatilisation d'une partie de l'azote sous forme de $\mathrm{N}_{2}$ et $\mathrm{N}_{2} \mathrm{O}$ (cf. article sur les émissions gazeuses). Par ailleurs une partie de l'azote ammoniacal peut aussi se dégager sous forme de $\mathrm{NH}_{3}$ gazeux.

La figure 5 présente la proportion de l'azote excrété qui est conservé dans le bâtiment (non volatilisé) lorsque les animaux sont sur litière, en comparaison de celle obtenue lorsqu'ils sont sur caillebotis intégral. L'utilisation de litière profonde (en couche épaisse) entraîne des pertes importantes d'azote, qui sont dans la fourchette des chiffres proposés par le CORPEN (2003) : 57 $\pm 13 \%$ avec des litières de paille, $72 \pm$ $7 \%$ avec des litières de sciure. Ramonet et Robin (2002) ont montré que la litiè-

Figure 5. Proportion d'azote conservé (non volatilisé) dans des bâtiments où les animaux sont élevés sur caillebotis intégral ou sur litière (d'après 1 : Portejoie et al 2002 (sans le stockage) ; 2 : CORPEN 2003 (avec le stockage) ; 3 : Ramonet et Callarec 2005 ; 4 : Robin et al $2004 b ; 5$ : Ramonet et Robin 2002).

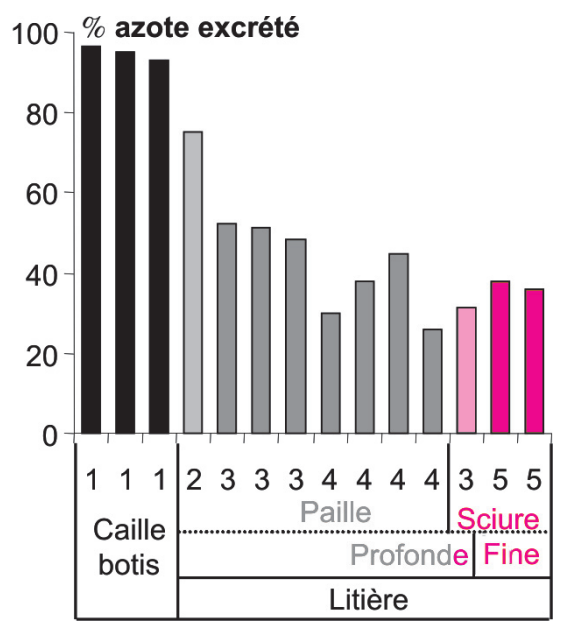

Figure 4. Répartition de l'azote ingéré et de l'azote excrété par des animaux recevant des régimes différant par leur teneur en protéines (d'après Portejoie et al 2004).

Répartition de l'azote ingéré (\%)

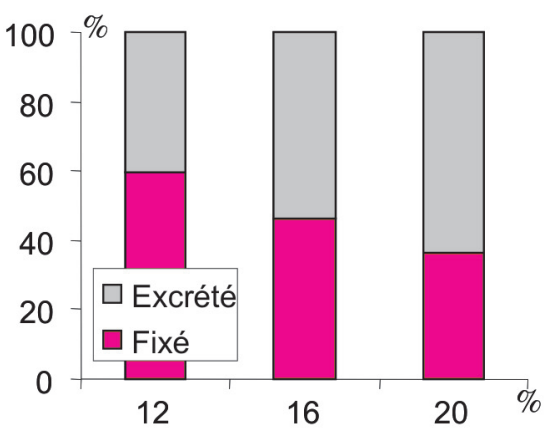

$\%$ protéines dans l'aliment
Répartition de l'azote excrété (\%)

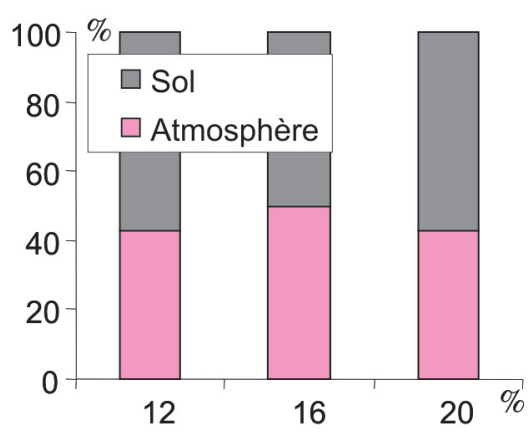

$\%$ protéines dans l'aliment 
re de sciure de bois en couche fine entraînait des pertes du même ordre de grandeur. Il faut noter cependant que la répartition de l'azote volatilisé suivant les différentes formes moléculaires $\left(\mathrm{N}_{2}, \mathrm{~N}_{2} \mathrm{O}\right.$ ou $\left.\mathrm{NH}_{3}\right)$ est très variable selon la conduite d'élevage et le type de litière (cf. article sur les émissions gazeuses).

Lors du stockage ultérieur des effluents, des quantités supplémentaires d'azote sont encore volatilisées, représentant 10 à $30 \%$ de l'azote sorti du bâtiment (CORPEN 2003, Espagnol et al 2006). Les pertes totales d'azote sont alors de l'ordre de $80 \%$ de l'azote excrété avec une litière de sciure et de $60 \%$ avec une litière de paille contre $25-30 \%$ avec du lisier.

\section{3 / Les pertes d'azote lors du compostage du lisier peuvent varier fortement selon les tech- niques utilisées et la nature des substrats}

Les pertes d'azote observées lors du compostage d'effluents porcins sont comprises entre 17 et $52 \%$ de l'azote initial : 32 à $52 \%$ pour Paillat et al (2005a) en conditions de fortes températures; 17 à $28 \%$ pour Espagnol et al (2006) sous faibles températures. Cet abattement intervient principalement dans la phase thermophile du compostage, pendant les deux premiers mois (cf. l'article sur les émissions gazeuses).

Une méthode de compostage sur paille a été développée à la station expérimentale de la Chambre d'Agriculture de Bretagne (méthode «Guernevez»). Elle consiste à imprégner de lisier en plusieurs fois de la paille maintenue en couche relativement fine. Comparativement aux techniques de compostage classique, elle permet de traiter des quantités plus élevées de lisier par tonne de paille utilisée comme substrat et met en œuvre plutôt le processus de nitrification/dénitrification, en raison des faibles températures atteintes $\left(<45^{\circ} \mathrm{C}\right)$. Les pertes d'azote sont comprises entre 50 et $75 \%$ (EDE Bretagne 1998). Lors de l'expérimentation conduite dans le cadre du programme «Porcherie verte», qui visait à quantifier les émissions gazeuses résultant de ce processus (cf. l'article sur les émissions gazeuses), l'abattement d'azote à été de $50 \%$ à la fin de la phase d'imprégnation $(70 \mathrm{j})$ et de $60 \%$ en fin de période de maturation (182 j).
Figure 6. Proportion d'azote volatilisé lors du compostage de fumier de dinde dans des conditions variables de porosité à l'air (d'après Paillat et al 2005a).

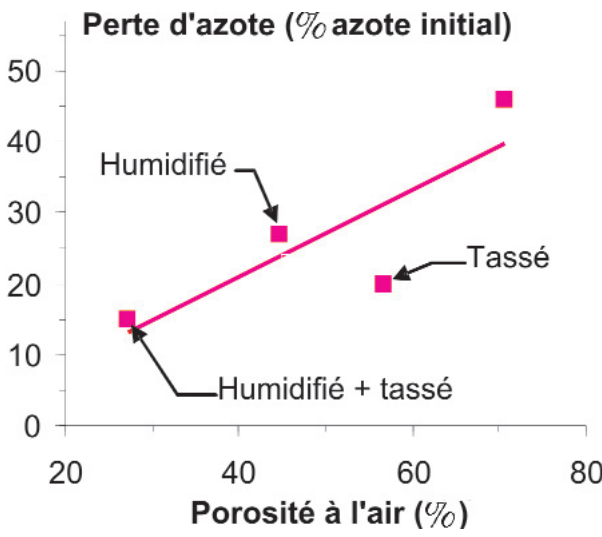

Abd El Kader et al (2007) ont montré, à partir d'une expérimentation sur fumier de dinde, que les conditions d'aération de l'andain influencent fortement les quantités d'azote éliminées par voie gazeuse, qui sont d'autant plus importantes que l'andain est plus aéré (figure 6). Paillat et al (2005b) ont montré aussi que les émissions d'ammoniac sont d'autant plus faibles que la quantité de carbone disponible, relativement à l'azote, est élevée (cf. l'article sur les émissions gazeuses). En fonction de ses objectifs agronomiques, l'exploitant agricole pourra ainsi ajuster les conditions dans lesquels il opère le compostage de ses effluents d'élevage :

- si l'objectif est de conserver l'azote dans le compost, il faut veiller à apporter suffisamment de carbone disponible, le rapport $\mathrm{C} / \mathrm{N}$ étant un critère clé de l'organisation de l'azote. Dans le cas des effluents d'élevage avec des rapports $\mathrm{C} / \mathrm{N}$ faibles, la source de carbone apporté doit être très biodégradable. Pour des fumiers très secs, l'ajout d'eau permet de mieux conserver le carbone et favorisera donc l'organisation de l'azote, mais un excès d'eau favorise la dénitrification et donc la perte d'azote. Pour des fumiers humides, le tassement est à éviter car, même s'il réduit la volatilisation d'ammoniac, il favorise la dénitrification. Un compromis entre ces processus est donc à rechercher ;

- si l'objectif est de traiter un effluent en éliminant de l'azote sous forme non polluante, un compostage en condition très humide permet une dénitrification à condition de favoriser la nitrification dans la couche supérieure aérée. Le compostage en couche de faible épaisseur (type méthode «Guernevez») est favorable à la conduite simultanée de ces processus antagonistes. Il nécessite de disposer de suffisamment de carbone biodégradable sinon, il existe un risque important de dénitrification incomplète avec émission de $\mathrm{N}_{2} \mathrm{O}$ (cf. l'article sur les émissions gazeuses).

\section{4 / Le traitement biologique abat la majeure partie de l'azote et certains types de stations per- mettent de capter le reste dans des coproduits potentiellement exportables}

Béline et al (2003) ont comparé les performances des principaux types de station de traitement biologique des effluents (figure 7). Elles permettent toutes d'abattre entre 60 et $70 \%$ de l'azote entrant qui est volatilisé (cf. l'article sur les émissions gazeuses). De plus, les stations de types 3 (séparation de phases par centrifugeuse) et de type 4 (séparation de phases du lisier brut par vis compacteuse et du liquide sortant du réacteur par un filtre à bande sous vide) captent l'essentiel des $30 \%$ d'azote non abattus dans des fractions suffisamment riches en solides pour qu'il soit économiquement faisable de les exporter. Ces fractions peuvent cependant être fortement chargées en cuivre et en zinc, ce qui peut les rendre difficilement exportables dans la pratique compte tenu des contraintes règlementaires sur les concentrations en éléments traces métalliques dans les effluents épandables (tableau 1).

\section{5 / Une meilleure connaissan- ce de la valeur fertilisante azotée à court terme des effluents, par une approche typologique}

Morvan et al $(2004,2006)$ ont établi une typologie des effluents à partir de l'étude de 47 produits de nature très variée (lisiers, fumiers, composts, coproduits de traitement...) caractérisés sur le plan biochimique et de leur dynamique de minéralisation du carbone et de l'azote dans le sol. L'objectif de la typologie a été de simplifier l'approche de la très grande diversité de composition et de réactivité des produits, en regroupant des produits $a$ priori différents. Les 47 produits étudiés ont été répartis en 6 classes qui présentent des dynamiques de minéralisation du carbone et de l'azote très différentes (figures 8 et 9), chacune de ces dynamiques correspondant à un profil de composition caractéristique (figure 9). 
Figure 7. Répartition des éléments à risques dans les différents coproduits issus de 4 types de station de traitement biologique (d'après Béline et al $2003 ; R S$ : Refus de séparation du lisier brut ; BB : boues biologiques ; SU : Surnageant ; Les fractions exportables en dehors de l'exploitation sont marquées d'un E; Pour le type 3, les fractions BB et SU sont confondues et représentées en noir plein).

\begin{tabular}{lcccc}
\hline Eléments de la station & Type 1 & Type 2 & Type 3 & Type 4 \\
\hline Bassin de stockage et d'homogénéisation & + & + & + & + \\
Séparateur de phases sur lisier brut* & & Vis & Centri & Vis \\
Réacteur biologique & + & + & + & + \\
Décanteur & + & + & + & \\
Séparateur de phases en sortie du réacteur & & & & + \\
\hline
\end{tabular}

* Séparation de phases par vis compacteuse (Vis) ou par centrifugeuse (Centri).
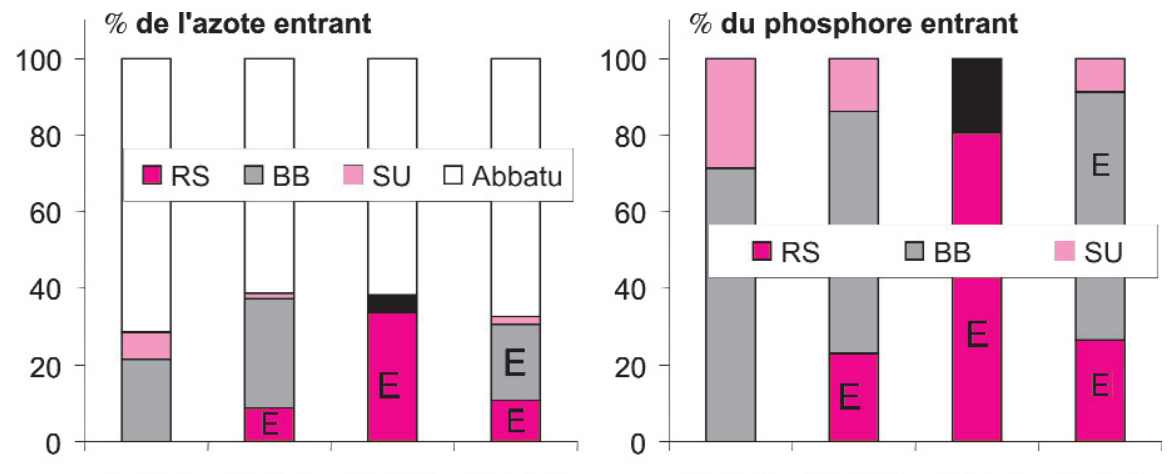

Type 1 Type 2 Type 3 Type 4

Type 1 Type 2 Type 3 Type 4

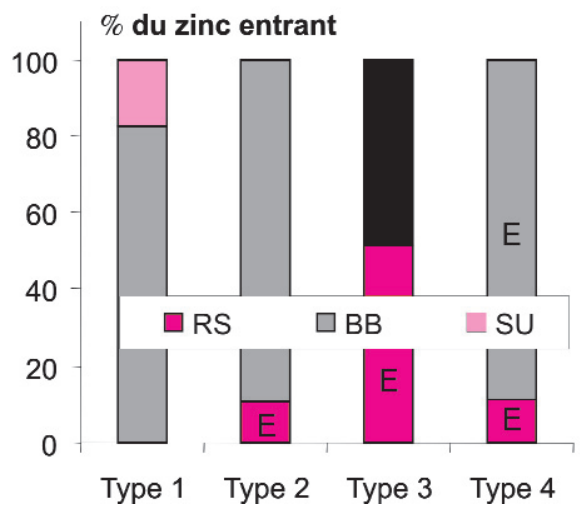

Figure 8. Dynamique de minéralisation du carbone et de minéralisation/organisation de l'azote de six classes d'effluents issues de la typologie de 47 produits variés (d'après Morvan et al 2004 ; mesures en conditions contrôlées de température $\left(15^{\circ} \mathrm{C}\right)$ et d'humidité [capacité au champ] pendant $224 \mathrm{j}$, sur un sol limoneux faiblement acide).
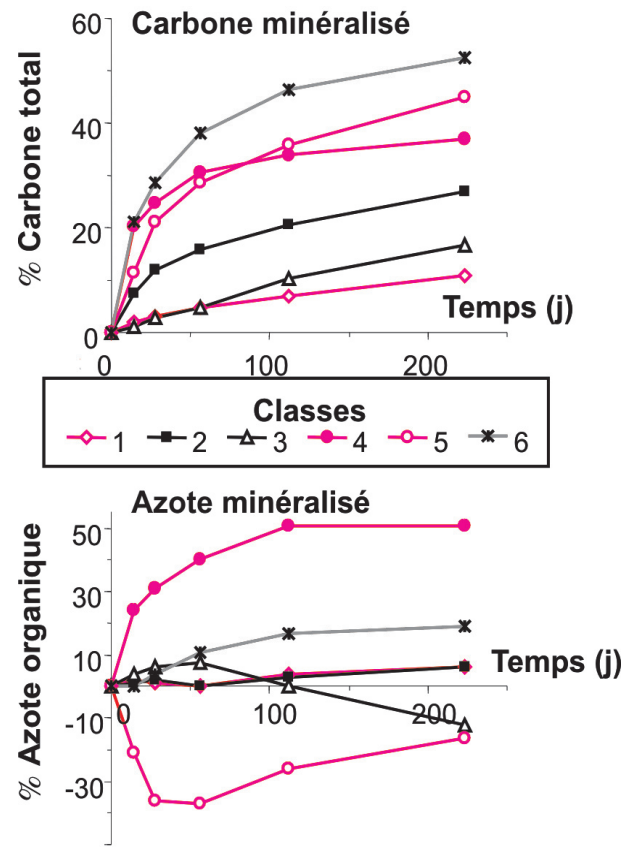

Azote organisé

Tableau 1. Principales caractéristiques des coproduits issus du traitement biologique des lisiers de porcs (d'après Béline et al 2003, cf. aussi figure 7).

\begin{tabular}{|ll|c|c|ccc|}
\hline Filières & & Type 2 & Type 3 & \multicolumn{3}{|c|}{ Type 4 } \\
\hline Coproduits & & RS $^{*}$ & RS & RS & BB $^{*}$ & RS + BB \\
\hline Matières sèches & - kg/tonne & 380 & 324 & 336 & 129 & 186 \\
\hline Matières organiques & - kg/tonne & 333 & 248 & 285 & 78 & 135 \\
& - g/kgMS & 868 & 765 & 848 & 604 & 726 \\
\hline Azote total & - kgN/tonne & 7,3 & 10,9 & 8,2 & 6,2 & 6,8 \\
& - gN/kgMS & 19,3 & 33,6 & 24,5 & 48,0 & 36,3 \\
Azote ammoniacal & - kgN/tonne & 2,7 & 4,4 & 3,5 & 0,1 & 1,1 \\
\hline Phosphore & - kgP/tonne & 5,4 & 9,1 & 5,9 & 6,6 & 6,4 \\
& - gP/kgMS & 14,2 & 28,1 & 17,4 & 50,8 & 34,2 \\
\hline Potassium & - kgK/tonne & 2,5 & 2,4 & 2,3 & 2,3 & 2,3 \\
& - gK/kgMS & 6,6 & 7,4 & 6,8 & 17,8 & 12,4 \\
\hline Cuivre** & - g/tonne & 54 & 96 & 48 & 140 & 114 \\
& - g/kgMS & 0,14 & 0,30 & 0,14 & 1,08 & 0,61 \\
\hline Zinc** & - g/tonne & 119 & 194 & 109 & 342 & 277 \\
& - g/kgMS & 0,31 & 0,60 & 0,32 & 2,65 & 1,49 \\
\hline Ratio N:P & & $1: 0,74$ & $1: 0,84$ & $1: 0,71$ & $1: 1,06$ & $1: 0,94$ \\
Ratio N:K & & $1: 0,34$ & $1: 0,22$ & $1: 0,28$ & $1: 0,37$ & $1: 0,34$ \\
\hline
\end{tabular}

* RS : refus de séparation, phase solide issue de la séparation du lisier brut

BB: boues biologiques, phase solide, pâteuse ou liquide issue de la séparation du lisier aéré

** La valeur en gras rouge dépasse le seuil fixé par l'arrêté sur les boues du 08/01/1998 (1 g Cu/kg MS et $3 \mathrm{~g} \mathrm{Zn} / \mathrm{kg} \mathrm{MS}$ ). Les valeurs de zinc et de cuivre sont toutes au dessus ou très proches des seuils fixés par la norme NF U 44-051 et les différents labels. 
Figure 9. Critères de composition permettant de définir l'appartenance d'un produit à une classe et relation entre ces profils et le comportement dans le sol, au terme de l'incubation $\left(224 j\right.$ à $\left.15^{\circ} \mathrm{C}\right)$ (d'après Morvan et al, 2006 ; C : carbone ; $S O L_{20}$ : soluble à l'eau à $20^{\circ} \mathrm{C}$; $S O L_{100}$ : soluble à l'eau à $100^{\circ} \mathrm{C}$ SOLVS : soluble après extraction selon Van Soest (1963) ; HEM : hémicellulose ; CEL : cellulose ; LIG : lignine ; --, $0,+,++$ : valeur très inférieure, inférieure, similaire, supérieure, très supérieure à la moyenne générale).

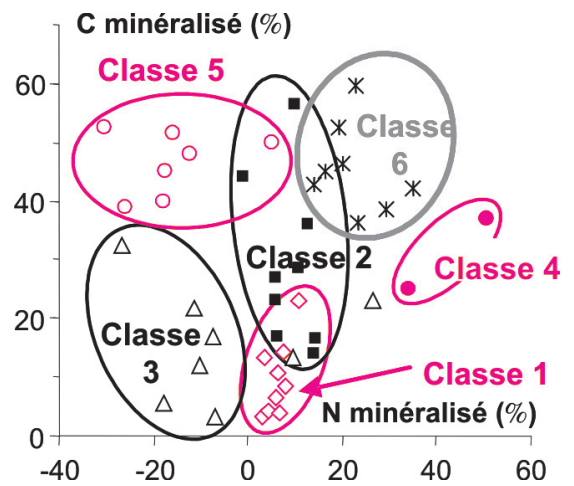

\begin{tabular}{|c|c|c|c|c|c|c|}
\hline Classe & $\begin{array}{l}5 \\
0\end{array}$ & $\begin{array}{l}3 \\
\triangle\end{array}$ & $\begin{array}{l}1 \\
0\end{array}$ & 2 & $\begin{array}{l}6 \\
*\end{array}$ & 4 \\
\hline Teneur C & + & + & -- & 0 & 0 & 0 \\
\hline \multicolumn{7}{|c|}{$\% C$ dans les fractions } \\
\hline $\mathrm{SOL}_{20}$ & + & - & -- & 0 & ++ & + \\
\hline $\mathrm{SOL}_{100}$ & - & -- & -- & 0 & + & ++ \\
\hline SOLvs & -- & -- & + & + & + & ++ \\
\hline HEM & ++ & 0 & - & $\mathbf{0}$ & 0 & 0 \\
\hline CEL & + & ++ & - & 0 & 0 & -- \\
\hline LIG & - & + & + & - & -- & -- \\
\hline
\end{tabular}

$\% \mathrm{~N}$ dans les fractions

$\mathrm{SOL}_{100} \quad++\quad 0 \quad--\quad 0 \quad++\quad++$

Les produits des classes 3 et 5 présentent une organisation nette de l'azote au cours de leur première année dans le sol, à mettre en relation avec la richesse de ces produits en cellulose (classes 3 et 5 ) et hémicelluloses (classe 5) et la faible solubilité de leur carbone. Ces produits ont donc une valeur fertilisante azotée nulle dans la première année qui suit leur épandage. Les produits de la classe 3 qui présentent une faible minéralisation du carbone comprennent l'ensemble des litières porcines sur déchets de bois ou paille de lin ainsi que les trois composts de lisier sur sciure (tableau 2). La classe 5, caractérisée par une forte biodégradation de la matière organique, regroupe le fumier de porc frais, trois des cinq lisiers compostés sur paille et trois des

Tableau 2. Répartition des 47 effluents d'élevage au sein des 6 classes issues d'une typologie basée sur la composition biochimique établie pour prédire la dynamique de minéralisation (d'après Morvan et al 2006, cf. aussi figures 8 et 9).

\begin{tabular}{|c|c|c|c|c|c|c|c|}
\hline \multirow[b]{2}{*}{ Produits } & \multirow{2}{*}{$\begin{array}{c}\text { Effectif } \\
\text { total }\end{array}$} & \multicolumn{6}{|c|}{ Répartition dans les classes } \\
\hline & & $\begin{array}{l}5 \\
0\end{array}$ & $\begin{array}{l}3 \\
\triangle\end{array}$ & & 2 & & 4 \\
\hline $\begin{array}{l}\text { Produits non traités } \\
\text { Lisiers de porc } \\
\text { Litières de volailles } \\
\text { Litières de copeaux ou de sciure } \\
\text { Fumier de porc } \\
\text { Fumiers de bovin }\end{array}$ & $\begin{array}{l}5 \\
2 \\
2 \\
1 \\
7\end{array}$ & 1 & 2 & & 7 & $\begin{array}{l}5 \\
2\end{array}$ & \\
\hline $\begin{array}{l}\text { Composts } \\
\text { Composts de fumier de bovin } \\
\text { Composts de litière de porc } \\
\text { Compost lisier porc + sciure } \\
\text { Compost lisier porc + paille } \\
\text { Compost lisier porc + matériaux carbonés }{ }^{* *} \\
\text { Compost lisier volailles + déchets verts } \\
\text { Compost de fumier de porc }\end{array}$ & $\begin{array}{l}2 \\
5 \\
3 \\
5 \\
6 \\
1 \\
1\end{array}$ & 3 & $\begin{array}{l}5 \\
3\end{array}$ & $\begin{array}{l}2 \\
6 \\
1 \\
1 \\
\end{array}$ & 2 & & \\
\hline $\begin{array}{l}\text { Coproduits de station de traitement biologique } \\
\text { Refus solide de séparation } \\
\text { Boues }\end{array}$ & $\begin{array}{l}4 \\
3 \\
\end{array}$ & 3 & & 1 & & 1 & 2 \\
\hline
\end{tabular}

* litière à base de sciure, écorce et/ou paille de lin.

** mélanges comportant des quantités variables d'écorces, de sciure, de paille, de déchets verts et/ou d'algues vertes.

quatre refus de séparation issus des stations de traitement biologique.

A l'inverse les produits des classes 4 et 6 présentent une minéralisation nette de l'azote au cours de leur première année dans le sol. Les produits de la classe 4 qui présentent en outre des teneurs en cellulose très faibles minéralisent leur azote plus rapidement que ceux de la classe 6. Ces deux classes de produits ont une valeur fertilisante azotée significative au cours de la première année après l'épandage, représentant de 25 à $50 \%$ de l'azote organique initial. Elles rassemblent les boues de station de traitement biologique et les lisiers frais de porc ou de volailles.

Les classes 1 et 2 se caractérisent par une minéralisation nette proche de zéro. La classe 1 regroupe des composts ayant fait l'objet d'une phase de maturation relativement longue (composts de lisier de porc sur paille ou déchets verts...) conduisant logiquement à une faible biodégradation de ces produits dans le sol. La classe 2 regroupe des fumiers de bovins stockés ou compostés, dont la biodégradation est plus importante, mais dont il résulte une faible minéralisation nette de l'azote.

La valeur fertilisante azotée faible ou nulle de la fraction organique des fumiers et composts pendant la 1ère année après l'épandage ne signifie pas qu'ils n'ont pas d'intérêt agrono- mique. Leurs effets se font sentir à plus long terme.

Les résultats de cette typologie seront très utiles pour la construction de modèles de la dynamique de minéralisation de l'azote apporté par les effluents d'élevage. Ces modèles permettront de prédire les quantités d'azote disponible pour les cultures en fonction du temps écoulé depuis l'application, et ainsi de mieux appréhender la valeur fertilisante azotée des effluents.

\section{2 / Le phosphore}

Les termes du bilan du phosphore dans une exploitation avec un atelier de production porcine s'établissent de façon plus simple que pour l'azote, du fait qu'il n'y a pas de formes d'émission gazeuse de cet élément. On peut suivre sur la figure 1, établie pour l'azote, les chemins possibles pour le cycle du phosphore en supprimant les rejets dans l'atmosphère et le lessivage.

Un porc en croissance finition fixe environ 5,5 g de phosphore par $\mathrm{kg}$ de gain (Jondreville et al 2004). Une partie du phosphore alimentaire, qui n'est pas digéré par l'animal, est excrétée dans les fèces et reste pour l'essentiel associée à la phase solide des effluents (figure 10). Si les apports de phosphore digestible dépassent les besoins des 
animaux, l'excès est majoritairement éliminé dans les urines, s'additionnant ainsi aux pertes endogènes de phosphore. Les traitements biologiques les plus simples et le compostage sont sans effet sur le phosphore dont seule la partie associée aux solides d'origine fécale peut être extraite des effluents par des procédés de séparation des phases solides et liquides.

\section{1 / La meilleure connaissance des besoins en phosphore des animaux permet d'ajuster les apports alimentaires en phos- phore digestible et donc de dimi- nuer la fraction excrétée dans les effluents}

Une connaissance précise des besoins des animaux en phosphore digestible permet d'ajuster au plus près les apports alimentaires et de réduire ainsi au minimum la quantité de phosphore digestible en excès (cf. figure 11).

\section{a) Porcs en croissance}

Sur la base des résultats obtenus au cours de 17 essais réalisés entre 1992 et 2001, Castaing et al (2003), ont estimé les besoins en phosphore digestible du porc en croissance (figure 11). Ils ont ainsi pu établir à 2,5 g par kg d'aliment les besoins en phosphore digestible chez des porcs en phase de croissance $(25-60 \mathrm{~kg})$ gagnant $770 \mathrm{~g}$ par jour. Le besoin n'est plus que de 1,8-2,0 g par $\mathrm{kg}$ d'aliment chez les mêmes porcs en phase de finition (60-105 kg) gagnant 830 g par jour. Ces résultats sont assis sur une base statistique solide, mais ils ne s'appliquent qu'aux situations moyennes décrites en phase de croissance (Gain Moyen Quotidien (GMQ) de $770 \mathrm{~g}$ par jour et Indice de Consommation (IC) de 2,3) et de fini-
Figure 11. Détermination des besoins en phosphore digestible de porcs en croissance pour optimiser la vitesse de croissance (GMQ) ou l'efficacité alimentaire (IC) en phase de croissance (en haut) ou de finition (en bas). Les données obtenues au cours de 17 essais ont été ajustées par un modèle linéaire-plateau. Le besoin en phosphore digestible se lit à la rupture de pente (d'après Castaing et al 2003, Jondreville et al 2005a).
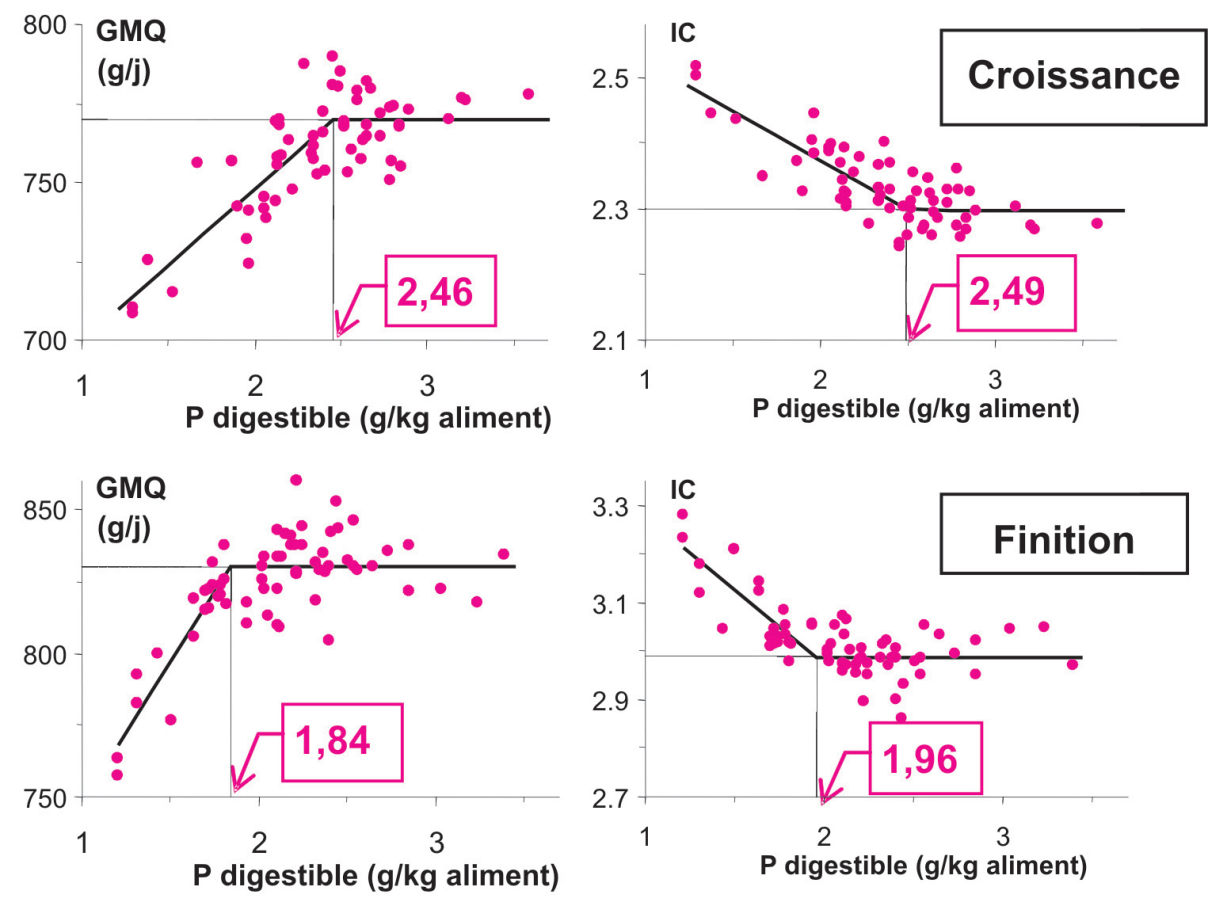

tion (GMQ de $830 \mathrm{~g}$ par jour et IC de 3,0$)$. Pour pouvoir prédire les besoins en phosphore digestible dans une situation quelconque, on peut les calculer selon une approche factorielle.

Jondreville et al (2005a) ont adapté l'approche factorielle pour calculer les besoins en phosphore digestible en fonction du stade de croissance et des performances des animaux, en faisant la somme du besoin d'entretien et du besoin de production. Dans ce cas, le besoin d'entretien, correspondant aux pertes endogènes urinaires uniquement,

est obtenu en multipliant le poids vif par $10^{-5}$ (d'après Guéguen et Perez 1981). Le besoin de production, correspondant à la fixation corporelle, est estimé à partir des courbes de contenu en phosphore corporel en fonction du poids vif (figure 12) obtenues par Jondreville et al (2004). Le calcul factoriel permet de retrouver, pour les deux cas moyens évoqués au paragraphe précédent, des résultats très proches de ceux obtenus par la méta-analyse d'un grand nombre d'essais (figure 11). Le calcul factoriel des besoins en phosphore digestible ainsi

Figure 10. Devenir du phosphore ingéré par le porc.

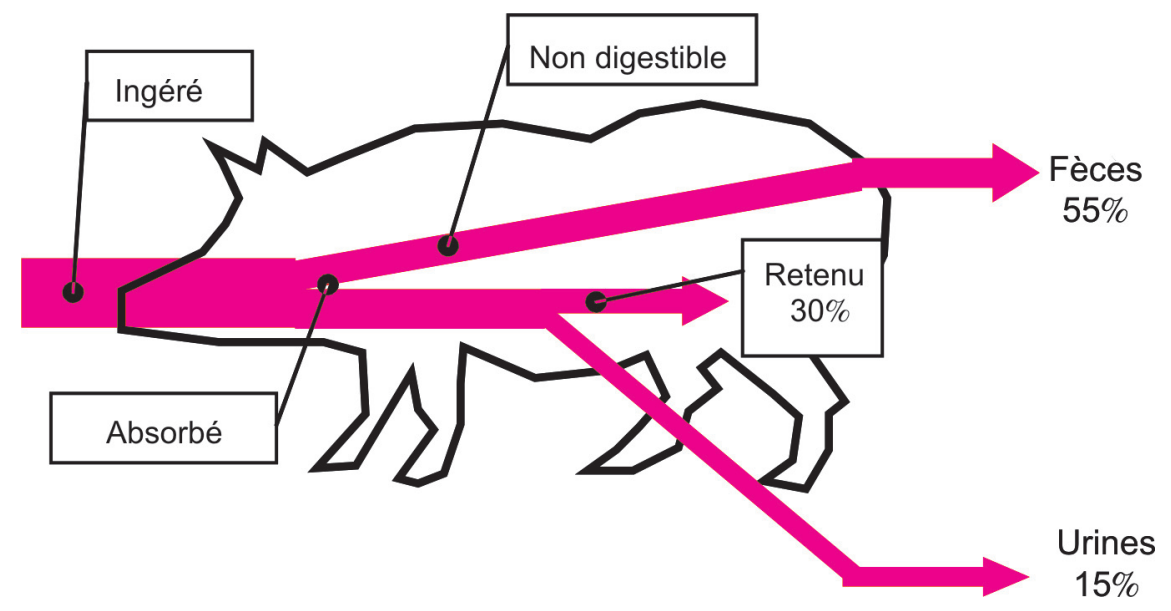
validé permet un ajustement précis des apports en phosphore digestible aux besoins des animaux dans toutes les situations d'élevage. On a ainsi les outils qui permettent d'éviter les excès de phosphore digestible qui se retrouvent dans les effluents (figure 10).

\section{b) Truies}

Cambeilh et al (2005) ont comparé trois niveaux d'apports alimentaires de phosphore digestible à des truies reproductrices $(2,6,2,3$ ou $2,0 \mathrm{~g} / \mathrm{kg}$ d'aliment en gestation ; 3,2, 2,9 ou 2,6 g/kg en lactation). L'originalité de l'étude est d'avoir été réalisée sur 5 cycles successifs de reproduction, ce qui permet de mettre en évidence d'éventuels effets à long terme. Une teneur en 
Figure 12. Evolution du contenu corporel en phosphore, zinc et cuivre chez le porc en croissance en fonction du Poids Vif Vide (PVV) (d'après Jondreville et al 2004).
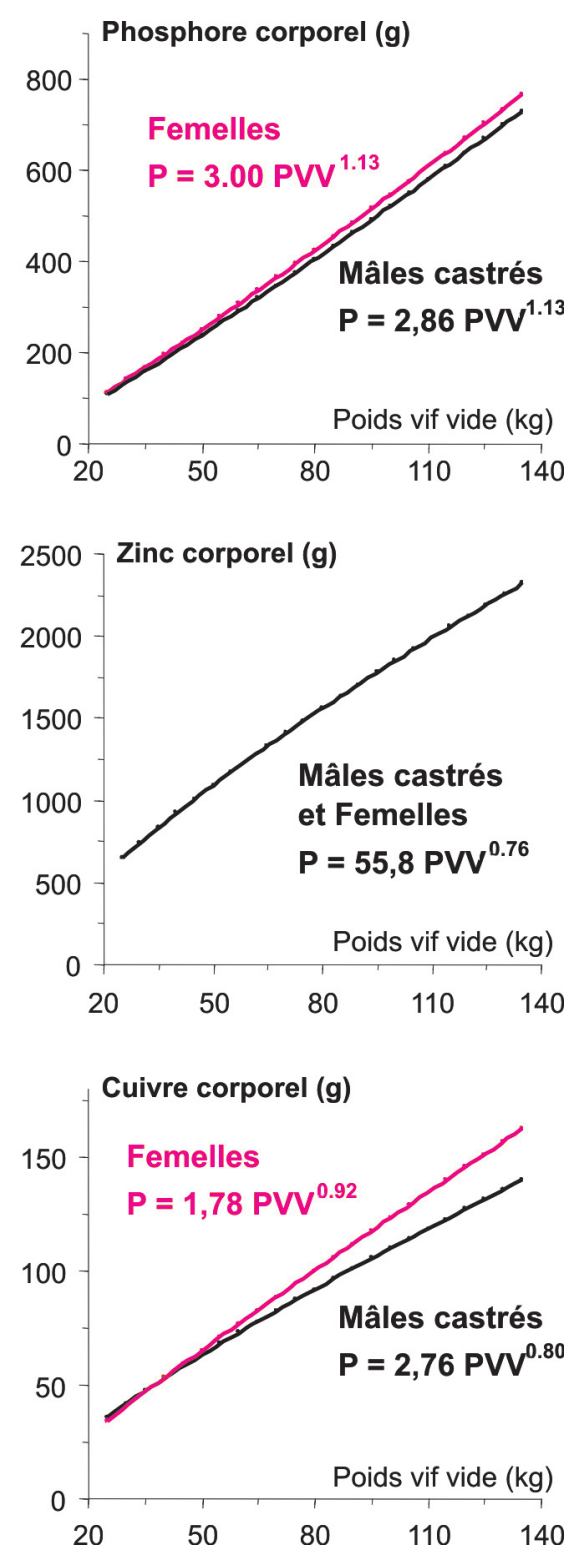

phosphore digestible de $2,3 \mathrm{~g} / \mathrm{kg}$ en gestation suivie de $2,9 \mathrm{~g} / \mathrm{kg}$ en lactation permet de garantir un bon niveau de productivité des truies, en bon accord avec les calculs factoriels des besoins proposés par l'INRA (Jondreville et Dourmad 2005). Les truies recevant des apports en phosphore inférieurs $(2,0 \mathrm{~g} / \mathrm{kg}$ en gestation et $2,6 \mathrm{~g} / \mathrm{kg}$ en lactation) ont eu un peu plus de problèmes locomoteurs et l'intervalle de temps entre le sevrage et la saillie fécondante du cycle de reproduction suivant a été légèrement accru, ce qui a entraîné une baisse de 0,8 porcelet produit par truie et par an. Ces apports bas peuvent cependant être envisagés si, au stade jeune reproduc- teur, l'aliment est suffisamment pourvu en phosphore.

La diminution des apports de 2,6 à 2,0 g de phosphore digestible par $\mathrm{kg}$ d'aliment en gestation et de 3,2 à 2,6 $\mathrm{g} / \mathrm{kg}$ lactation permet de réduire de $23 \%$ les rejets de phosphore des truies (figure 13), de 4,0 à $3,1 \mathrm{~kg}$ de phosphore par truie et par an. L'économie réalisée est plus modeste $(10 \%)$ avec le régime intermédiaire (2,3 $\mathrm{g} / \mathrm{kg}$ en gestation, $2,9 \mathrm{~g} / \mathrm{kg}$ en lactation) plus sécurisant du point de vue des performances et de la santé des animaux.

Figure 13. Bilan global du phosphore alimentaire de trois groupes de truies recevant des apports différents de phosphore en gestation et en lactation (bilan cumulé sur 5 cycles de reproduction, d'après Cambeilh et al 2005).

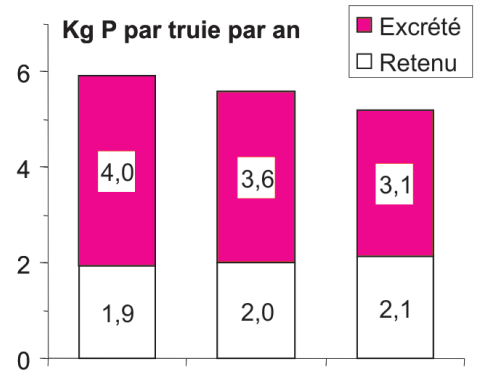

Apports alimentaires de $\mathbf{P}$ ( $\mathbf{g} / \mathbf{k g}$ aliment) Gestation 2,6 2,3 2,0 Lactation $\quad 3,2 \quad 2,9 \quad 2,6$

\section{2 / Une meilleure digestibilité du phosphore alimentaire se tra- duit par une diminution de la quantité excrétée}

Dans les aliments destinés au porc, le phosphore est présent sous diverses formes. Les phosphates présentent une digestibilité variable (Sauvant et al 2002), atteignant $66 \%$ pour le phosphate bicalcique anhydre à $83 \%$ pour le phosphate monocalcique (Jondreville et Dourmad 2005). Dans les matières premières végétales (céréales, protéagineux et tourteaux), 50 à $80 \%$ du phosphore est présent sous forme d'acide phytique. Cette forme de phosphore est très peu valorisée par les animaux monogastriques qui ne disposent pas de l'équipement enzymatique nécessaire à la déphosphorylation des phytates. Les matières premières végétales contiennent par ailleurs, en quantité également très variables, des phytases endogènes capables de dégrader les phytates, rendant ainsi le phosphore accessible à la digestion par l'animal. Enfin, il est possible d'ajouter à l'aliment de la phytase exogène d'origine microbienne. Ainsi, la digestibilité du phosphore dans un aliment porc dépendra :

- de la digestibilité du phosphore dans les matières premières constitutives de l'aliment, en lien avec la proportion de phosphore sous forme phytique et le niveau de l'activité phytasique endogène ;

- de la supplémentation ou non de l'aliment en phytase exogène ;

- du type de phosphate ajouté.

L'amélioration de la digestibilité du phosphore alimentaire, couplée à une bonne connaissance des besoins des animaux (cf. supra), permet de réduire au minimum les ajouts de phosphore dans les compléments minéraux, et de diminuer ainsi les rejets de phosphore dans les effluents.

a) La digestibilité du phosphore dans les matières premières alimentaires dépend de la proportion de phosphore sous forme de phytates et de l'activité phytasique endogène

Skiba et al (2004) ont mesuré la digestibilité du phosphore dans six familles de matières premières caractérisées par leur teneur en phosphore total et phytique et le niveau de l'activité phytasique endogène. La digestibilité varie fortement entre familles (figure 14).

Lorsque la proportion de phosphore phytique est élevée (autour de 60\%), comme c'est le cas pour les céréales, la digestibilité du phosphore est dépendante du niveau d'activité de la phytase endogène. C'est ainsi que l'orge, et plus encore le maïs, présentent une digestibilité du phosphore inférieure à celle observée pour le blé ou le triticale. Les variétés de maïs à faibles teneurs en phosphore phytique présentent logiquement une bonne digestibilité du phosphore, en dépit du faible niveau d'activité phytasique. La bonne digestibilité du phosphore chez le pois résulte de la combinaison d'une proportion de phosphore phytique inférieure à celle des céréales et d'une activité phytasique intermédiaire entre celles de l'orge et du maïs.

b) L'adjonction de phytase exogène à l'aliment permet d'améliorer la digestibilité du phosphore

L'adjonction aux aliments de phytase exogène d'origine microbienne permet d'améliorer la digestibilité du phosphore alimentaire. Différentes études permettent d'estimer que 500 unités de phytase microbienne (3-phytase 
Figure 14. Variations entre matières premières (blé, triticale, orge, maïs, maïs à faible teneur en phytate, pois) de la proportion de phosphore phytique, de l'activité phytasique et de la digestibilité du phosphore chez le porc en croissance (d'après Skiba et al 2004 ; Maïs LP : Maïs à faible teneur en phytates ; Moyennes \pm écart-type).
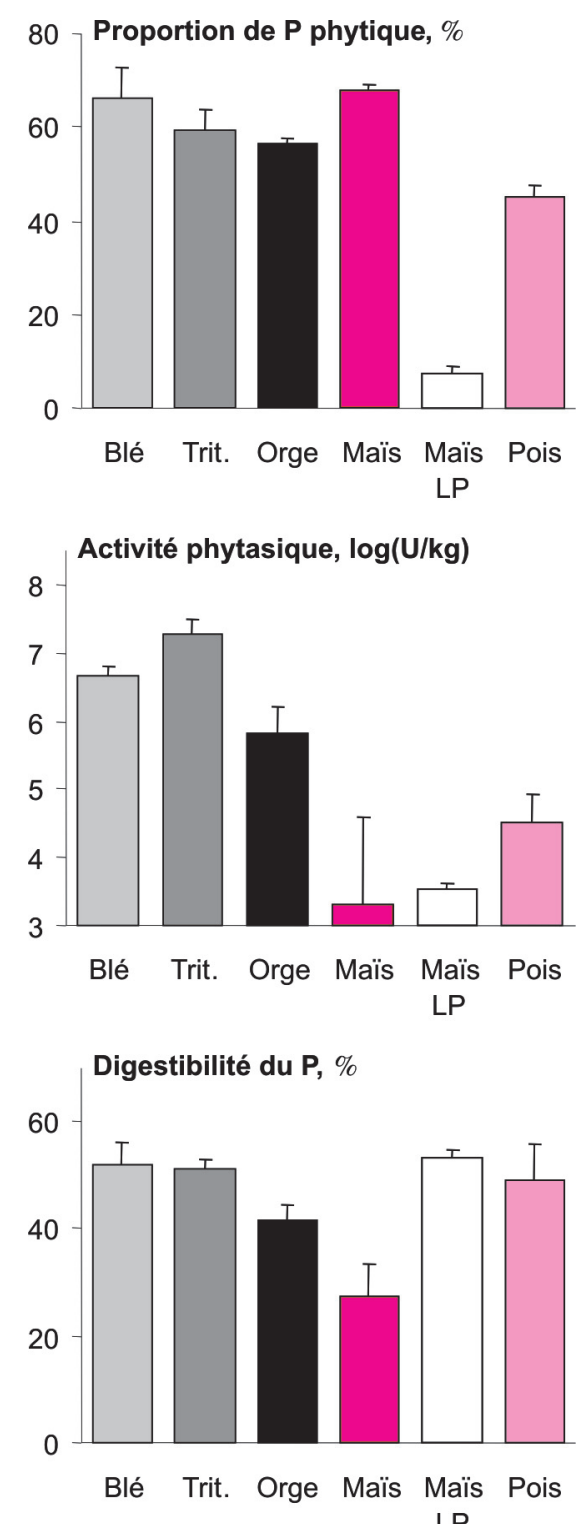

produite à partir d'Aspergillus niger) introduites dans un aliment à base de maïs et de tourteau de soja équivalent à 0,65-0,75 g de phosphore digestible (Jondreville et Dourmad 2005). Cet effet bénéfique a été confirmé dans les essais conduits par Jondreville (2003) et Revy et al (2004a et b) sur les effets de la phytase sur la disponibilité du zinc (cf. §3.2). L'adjonction de 800 UI de phytase microbienne a été équivalente à $0,89 \mathrm{~g}$ de phosphore digestible par kg d'aliment (figure 15), permettant ainsi de réduire sensiblement la part du phosphore alimentaire rejeté par l'animal.
Figure 15. Répartition du phosphore apporté par deux aliments supplémentés ou non en phytase exogène d'origine microbienne (d'après Jondreville 2003).

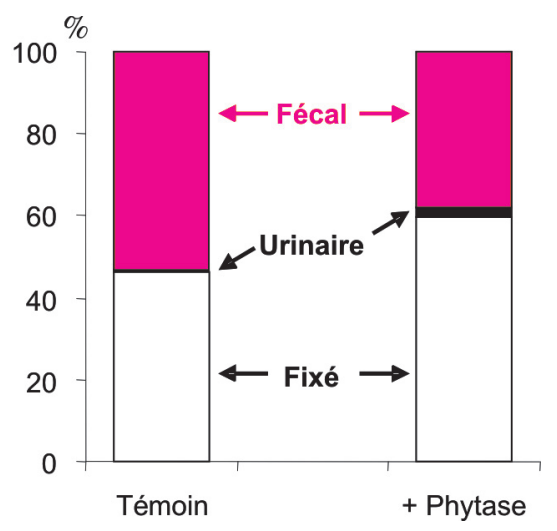

2.3 / Les traitements biologiques avec séparation de phases permettent de capter le phosphore dans des coproduits potentiellement exportables

Béline et al (2003) ont comparé les performances des principaux types de stations de traitement biologique des effluents (figure 7). Les stations qui ne sont pas équipées de séparateur de phases (type 1) laissent la totalité du phosphore dans les boues biologiques et le surnageant, qui doivent être épandus sur l'exploitation ou à proximité immédiate. Dans les stations de type 2, la vis compacteuse utilisée pour extraire la fraction solide des lisiers bruts ne sépare qu'un quart du phosphore entrant. Si une centrifugeuse remplace la vis compacteuse, plus de $80 \%$ du phosphore entrant est extrait dans des coproduits potentiellement exportables. Une séparation de phases intervenant après le réacteur biologique permet de porter le pourcentage d'extraction au-delà de $90 \%$. Les coproduits obtenus peuvent cependant être fortement chargés en cuivre et en zinc, ce qui peut les rendre difficilement exportables dans la pratique compte tenu des contraintes règlementaires sur les concentrations en éléments traces métalliques dans les effluents épandables (tableau 1).

\section{4 / La valeur fertilisante} phosphatée des effluents est en général très élevée et n'est pas corrélée à la spéciation du phosphore dans les produits

Les mesures de biodisponibilité du phosphore de 9 effluents d'élevage por- cin ont permis d'établir la Valeur Fertilisante Phosphatée (VFP) très élevée de la quasi-totalité des produits et coproduits étudiés (figure 16). La valeur fertilisante des litières, des boues et des composts est similaire à celle de l'engrais de référence. Elle est un peu plus faible (85 à $88 \%$ ) pour les refus de séparation de phase. La biodisponibilité n'apparaît pas corrélée à la spéciation du phosphore dans les produits (formes organiques et minérales). Sur le long terme, il est probable que la totalité du phosphore apporté contribue efficacement à la fertilité des sols.

Figure 16. Valeur fertilisante phosphatée (en \% de l'engrais de référence) de 9 effluents d'élevage porcin (d'après Morvan et al 2004, Linères et al 2005).

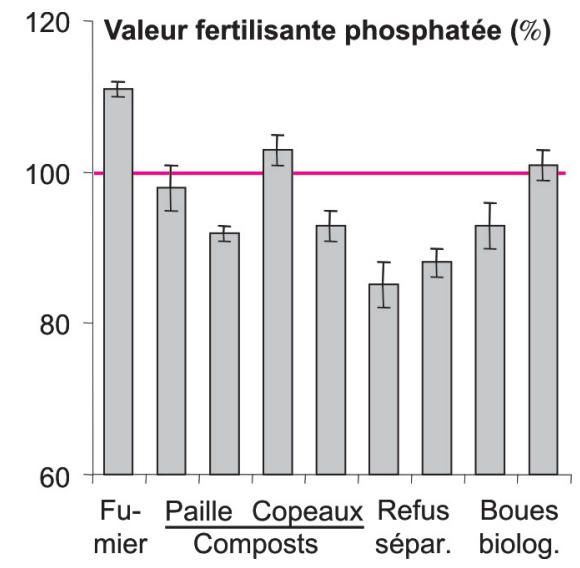

Les valeurs sont en moyenne plus élevées que celles obtenues par les mêmes auteurs pour des fumiers de bovins $(76 \%)$ ou des fientes de volailles $(87 \%)$ selon la même méthode. Les boues de traitement des lisiers ont les mêmes valeurs fertilisantes que les boues biologiques de station d'épuration urbaines non stabilisées par digestion ou compostage ( $92 \%$ en moyenne).

Compte tenu de ces résultats, les effluents porcins devraient être considérés comme des engrais phosphatés. Pour les produits étudiés dans cette action, des apports de 1 à 5 tonnes de matière sèche devraient suffire à couvrir les exportations annuelles d'une culture intensive. Dans le cas d'apports répétés sur une même parcelle il est raisonnable de prendre en compte la totalité du phosphore apporté. Dans le cas des lisiers traités dont le rapport N/P est faible, les apports de phosphore seront excessifs si la fertilisation est raisonnée uniquement en fonction des apports d'azote maxima autorisés. 


\section{3 / Les éléments traces métalliques (cuivre et zinc)}

Certains éléments métalliques comme le cuivre et le zinc, lorsqu'ils sont incorporés à l'aliment, améliorent les performances de croissance des animaux (Braude 1975). Leurs effets favorables, dont les mécanismes sont mal compris, passent par une amélioration de l'état sanitaire, en particulier pour ce qui concerne les pathologies digestives. Ce type de pathologies est fréquent en élevage porcin, à l'état clinique ou subclinique, notamment en phase de postsevrage, dès lors que les conditions d'ambiance sont sous-optimales (densité, pression microbienne...). Elles ont longtemps été combattues par une antibiothérapie préventive systématique, mais cette pratique est maintenant interdite. Les éleveurs sont donc incités à incorporer du cuivre et du zinc dans les aliments, à des doses qui dépassent largement les besoins alimentaires stricto sensu, qui permettent d'éviter les carences. Mais le cuivre et le zinc sont très peu absorbés par l'animal et l'essentiel de ce qui est ajouté dans les aliments est rejeté dans les effluents épandus. Ils s'accumulent de façon irréversible dans le sol où ils peuvent exercer une action phytotoxique dès lors que leur concentration dépasse un certain seuil. Les concentrations observées dans les sols bretons, qui reçoivent beaucoup d'effluents porcins, sont en constante augmentation (Coppenet et al 1993 ) et on peut craindre à terme une stérilisation des sols.

La généralisation du traitement des effluents ne fait qu'aggraver la situation. En effet, les traitements (compostage, traitement biologique sans séparation de phases...) utilisés pour abattre l'azote ont peu ou pas d'effet sur ces éléments. Les coproduits, appauvris en azote, peuvent être épandus en plus grande quantité à l'hectare que les lisiers bruts. A concentration initiale égale, les quantités d'éléments traces métalliques apportées au sol sont donc beaucoup plus élevées lorsque les effluents sont traités. Ainsi, ces quantités peuvent être 5 fois plus importantes après un traitement qui abat $80 \%$ de l'azote sans opérer de séparation des phases solides et liquides.

La maîtrise des rejets de cuivre et de zinc passe ainsi par une réduction des apports alimentaires. Une première étude conduite par Paboeuf et Jondreville (2005) a permis d'approcher les apports minimaux nécessaires au maintien des performances, dans des conditions d'ambiance sous-optimales proches de la réalité du terrain. Par ailleurs, Révy et al (2004a et b, 2006) se sont attachés à déterminer les besoins sensu stricto (prévention des carences), dans les conditions, plus favorables, d'un élevage expérimental.

\section{1 / Détermination des besoins des animaux}

a) La supplémentation par des éléments traces métalliques est utile pendant la phase de post-sevrage, mais pas au-delà

Paboeuf et Jondreville (2005) ont testé, au cours de trois expérimentations successives, la possibilité de réduite les teneurs en cuivre et en zinc par rapport aux pratiques courantes avant la réglementation de 2003 (figure 17 ). Selon ces pratiques, le cuivre est introduit dans les aliments à un niveau pharmacologique dans les premières phases de croissance $(>150 \mathrm{mg} / \mathrm{kg}$ d'aliment) alors que l'usage de zinc comme facteur de croissance est proscrit par la réglementation européenne, même en post-sevrage. Les résultats montrent que les animaux sont peu sensibles à une réduction drastique des teneurs en cuivre et en zinc pendant les périodes de croissance et de finition, au-delà de 40 j post-sevrage (expériences 1 et 2). Par contre, ils réagissent défavorablement à une réduction forte des teneurs en cuivre et en zinc pendant le mois qui suit le post-sevrage (expérience 3 ), par une réduction de la vitesse de croissance et une détérioration de l'efficacité alimentaire qui s'observe non pas pendant la phase de post-sevrage, mais au-delà de $40 \mathrm{j}$ (effet retard). Cette détérioration est probablement due à la diminution de l'apport alimentaire de cuivre en post-sevrage, puisque le zinc n'était pas apporté à des niveaux pharmacologiques.

Comme le préconise la nouvelle réglementation, il semble donc de bonne stratégie de supplémenter les aliments en cuivre pendant la phase de post-sevrage, à une période où les animaux y répondent bien et consomment assez peu car ils sont encore assez légers, mais de renoncer à supplémenter au-delà du besoin stricto sensu de prévention des carences au-delà de cette phase. Ainsi, l'aliment à teneur réduite de l'expérience 2 permet de réduite de $60 \%$ les rejets de cuivre et de $20 \%$ les rejets de zinc par rapport à l'aliment témoin de l'expérience 1 , sans effet négatif sur les performances des animaux (figure 17). b) Détermination des besoins strictosensu

Une première démarche pour déterminer les besoins consiste, à l'instar de ce qui a été fait pour le phosphore (cf. supra) à déterminer les quantités fixées par l'animal dans ses dépôts corporels (figure 12). En dérivant les courbes obtenues par Jondreville et al (2004), on calcule qu'un porcelet en post-sevrage fixe de l'ordre de 21-26 $\mathrm{mg}$ de zinc et 1,3-1,4 $\mathrm{mg}$ de cuivre par $\mathrm{kg}$ de gain de poids. Mais les besoins ne se limitent pas aux quantités fixées car ces éléments sont fortement impliqués dans les processus métaboliques de l'animal.

Sur la base de critères liés à l'utilisation métabolique du zinc (et donc ne prenant pas en compte les effets de prévention des pathologies digestives), Revy et al (2004a et b, 2006) ont montré, que le besoin en zinc stricto sensu (prévention des carences) du porcelet en post-sevrage est de l'ordre de 40-43 $\mathrm{mg} / \mathrm{j}$ soit environ $90 \mathrm{mg}$ par $\mathrm{kg}$ d'aliment (figure 18, données en noir), ce qui est proche du résultat obtenu par méta-analyse des résultats de la littérature (Jondreville et al 2003). Les quantités de zinc présentes dans les matières premières alimentaires sont en général insuffisantes pour couvrir ce besoin et il est nécessaire de complémenter par des apports de zinc sous une forme très disponible comme le sulfate de zinc. Dans le cas où le zinc complémentaire est apporté sous une forme moins disponible (par exemple sous forme d'oxyde), les apports doivent être revus à la hausse, avec une augmentation concomitante des quantités rejetées.

\section{2 / L'adjonction de phytase microbienne à l'aliment amélio- re la disponibilité du zinc pour l'animal}

Une partie du zinc présent dans les matières premières alimentaires est complexé à l'acide phytique. L'adjonction de phytase exogène d'origine microbienne dans les aliments pour porcs améliore très significativement la disponibilité du zinc présent dans les matières premières (Revy et al 2006, Jondreville et al 2005b ou c), mais est sans effet notable sur la disponibilité du cuivre (Jondreville 2003). Cette enzyme permet donc de réduire très sensiblement les apports de zinc (figure 18), de $90 \mathrm{mg}$ par $\mathrm{kg}$ d'aliment témoin à $50 \mathrm{mg}$ par $\mathrm{kg}$ d'aliment contenant 700 $\mathrm{U}$ de phytase exogène (3-phytase produite par Aspergillus niger). Ainsi, l'adjonction de $700 \mathrm{U}$ de cette 
Figure 17. Effets de la distribution d'aliments à teneurs variables en éléments trace métallique sur les performances de croissance et les quantités excrétées (d'après Paboeuf et Jondreville 2005 ; en noir : aliments témoins ; en rouge : aliments à teneurs réduites ; Les observations ont été réalisées au cours de 3 expériences successives).

Figure 17a. Teneurs des aliments en cuivre et en zinc en fonction du temps après le sevrage (périodes $1: 0-14 j ; 2: 15-39 j ; 3: 40-80 j ; 4: 81 j$ à l'abattage).
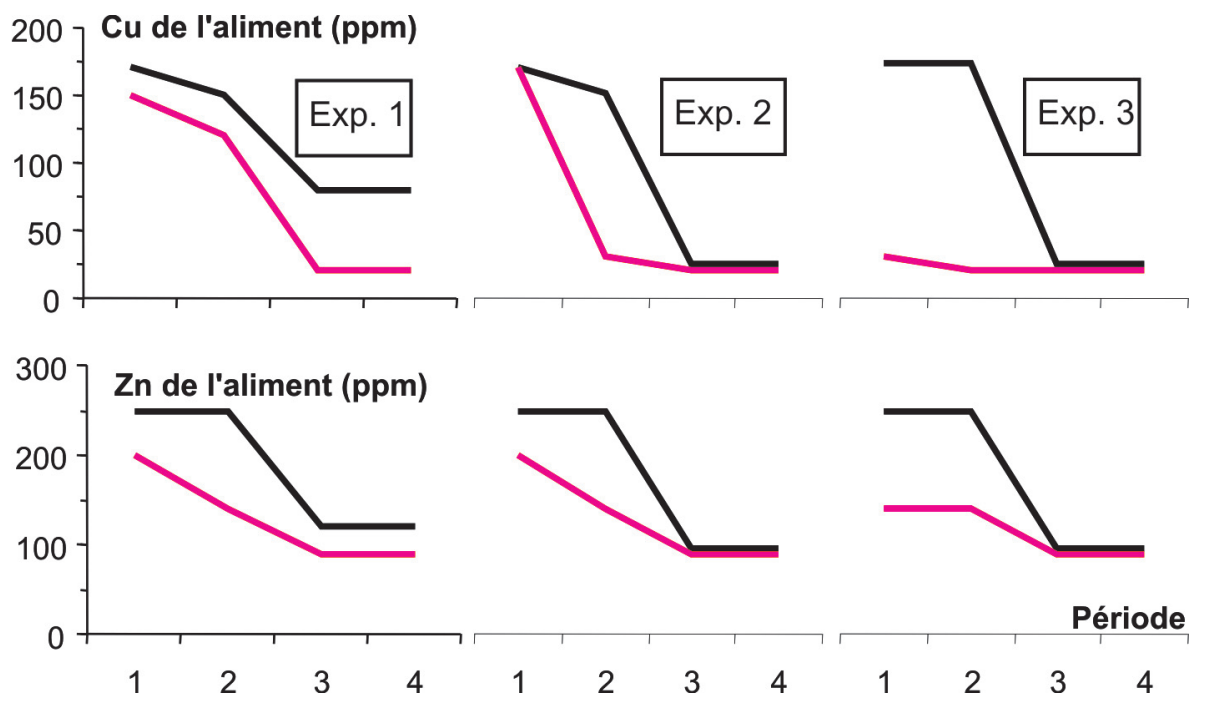

Figure 17b. Gain moyen quotidien (GMQ) et indice de consommation (kg aliment par kg de gain).

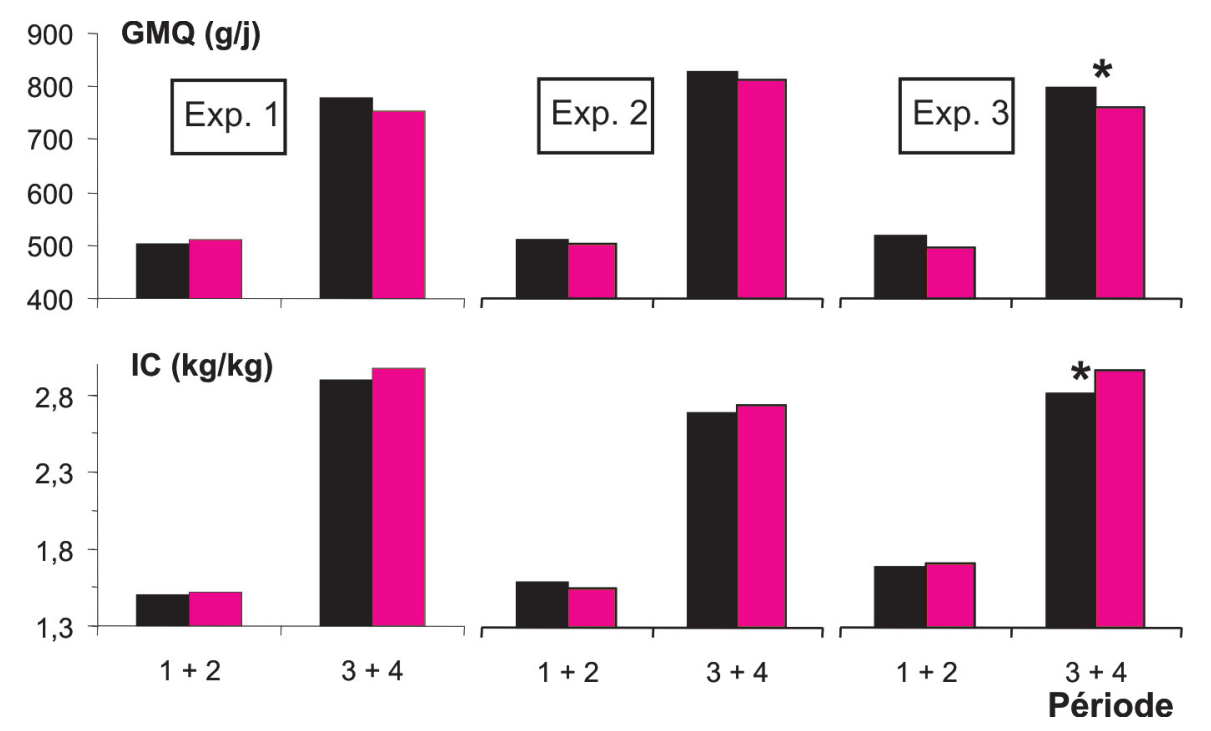

Figure 17c. Quantités totales de cuivre et de zinc excrétées par chaque animal.

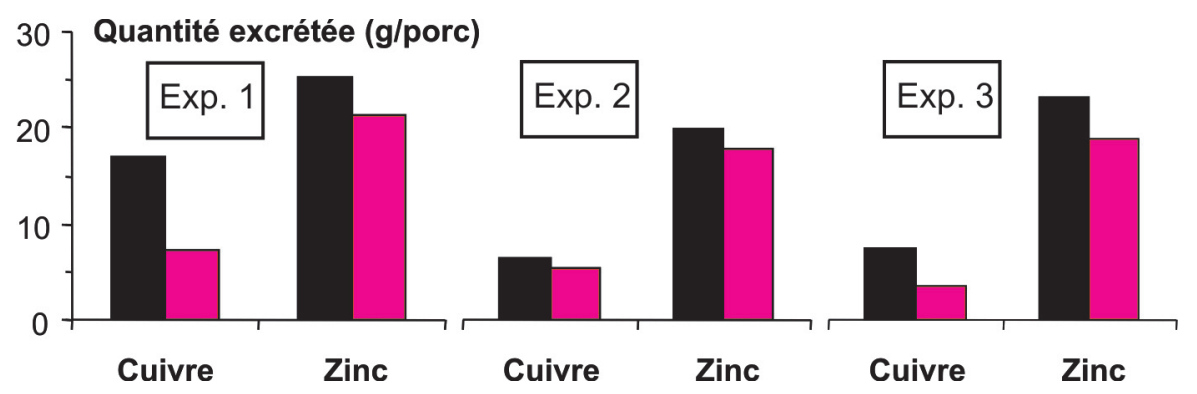

3-phytase permet d'épargner $40 \mathrm{mg}$ de zinc, ce qui correspond à une réduction des rejets de l'ordre de $40 \%$. La figure 19 permet de déterminer l'épargne de zinc en fonction de la quantité de phytase microbienne ajoutée dans l'aliment.

\section{3 / Les traitements biolo-} giques avec séparation de phases permettent de capter le zinc et le cuivre dans des coproduits potentiellement exportables

Béline et al (2003) ont comparé les performances des principaux types de station de traitement biologique des effluents (figure 7). Les stations qui ne sont pas équipées de séparateur de phases (type 1) laissent la totalité du zinc et du cuivre dans les boues biologiques et le surnageant, qui doivent être épandus sur l'exploitation ou à proximité immédiate (cf. infra, §3.5). Dans les stations de type 2, la vis compacteuse utilisée pour extraire la fraction solide des lisiers bruts ne sépare que $10 \%$ des éléments traces métalliques entrant. Si une centrifugeuse remplace la vis compacteuse, la moitié du zinc et un tiers du cuivre sont extrait dans des coproduits potentiellement exportables. Une séparation de phases intervenant après le réacteur biologique permet de capter la totalité des éléments traces métalliques entrant. Mais les fortes teneurs en zinc et en cuivre des coproduits peuvent les rendre difficilement exportables dans la pratique compte tenu des contraintes règlementaires sur les concentrations en éléments traces métalliques dans les effluents épandables (tableau 1).

3.4 / Après épandage, les éléments traces métalliques sont peu mobiles dans le sol

Jaffrézic et al (2005) ont déterminé sous quelles formes se trouvent le zinc et le cuivre dans neuf effluents variés. Les teneurs totales en zinc et en cuivre sont très variables d'un produit à l'autre $(0,29$ à $2,45 \mathrm{~g} / \mathrm{kg}$ produit sec pour le zinc et 0,19 à $1,18 \mathrm{~g} / \mathrm{kg}$ pour le cuivre). Les concentrations les plus faibles sont observées dans les litières et fumier ; elles sont élevées dans les lisiers et très élevées dans les boues biologiques. Une extraction séquentielle des effluents par des méthodes chimiques (spéciation) montre que les proportions d'éléments traces métalliques sous forme soluble ou échangeable sont plus élevées pour le zinc que pour le cuivre (figure 20). Le zinc est donc potentiellement plus mobile dans le sol. Le pourcentage de cuivre sous 
Figure 18. Détermination des besoins en zinc de porcs en croissance avec un aliment supplémenté (en rouge) ou non supplémenté (en noir) avec de la phytase exogène d'origine microbienne pour optimiser l'activité de la Phosphatase Alcaline Plasmatique (APA) ou la concentration plasmatique de zinc. Les données de l'essai ont été ajustées par un modèle linéaire-plateau. Le besoin en zinc se lit à la rupture de pente (d'après Revy et al 2004a, 2006).

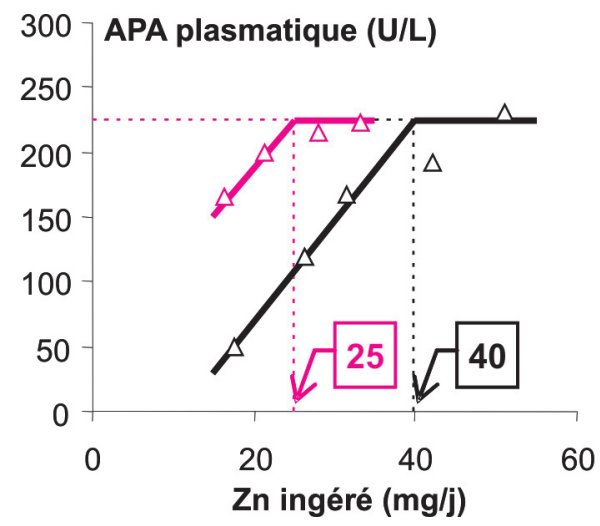

Figure 19. Epargne de zinc permise par l'adjonction de phytase microbienne (d'après Jondreville et al 2005b et c).

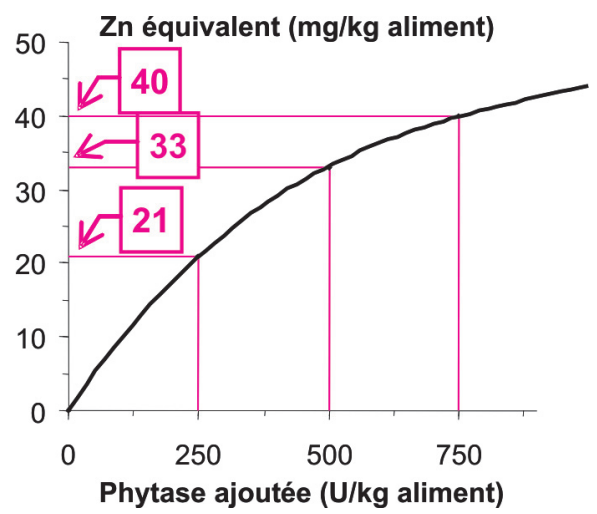

forme soluble ou facilement échangeable se révèle nettement plus élevé dans les produits compostés, comparativement aux lisiers ou aux boues biologiques, et doit être relié aux processus de minéralisation de la matière organique durant le compostage.

Cependant la mobilité réelle des éléments traces dans le sol après épandage ne peut être totalement appréhendée par les méthodes de spéciation, en raison de la complexité des mécanismes de solubilisation, complexation et adsorption et des interactions entre les matrices solubles du sol et du produit organique. La caractérisation des formes du cuivre dans le sol pendant plusieurs semaines après l'apport met en évidence une augmentation de courte durée des teneurs en cuivre soluble (figure 23). Le risque de transfert et de pollution est donc avéré mais faible et très localisé dans le temps, en raison des faibles pourcentages de cuivre mobilisé, et de la faible durée pendant

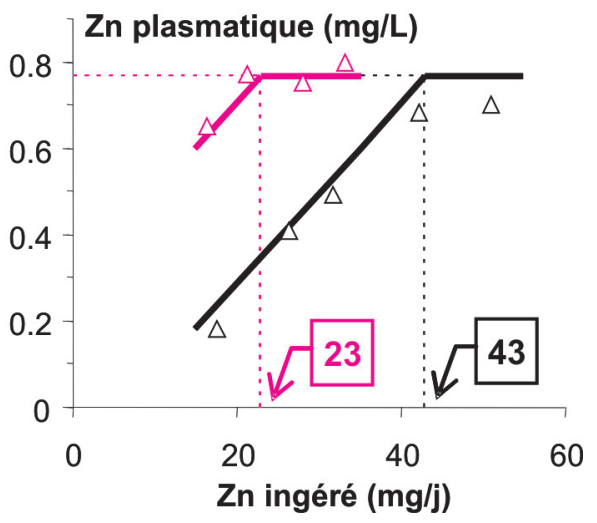

laquelle ce risque peut s'exprimer. Ces mesures confirment que les éléments traces métalliques apportés au sol y restent pour l'essentiel stockés sur des temps longs. al 2004).
3.5 / Les apports d'éléments traces métalliques au sol excèdent en général largement les capacités d'exportation par les plantes

Dourmad et Jondreville (2007) ont calculé les quantités d'éléments traces métalliques épandues sur le sol dans le cadre de plusieurs stratégies d'apport alimentaire de zinc et de cuivre, et les ont comparées aux capacités d'exportation par les plantes (200 g de zinc et $50 \mathrm{~g}$ de cuivre par ha et par an).

Pour le cuivre, les auteurs ont comparés les stratégies suivantes (figure 24a) :

- UE anc. : apports selon l'ancienne réglementation de l'UE, en vigueur jusqu'en 2003 ;

- UE : apports selon la réglementation actuelle de l'UE ;

- Besoin : apports de $10 \mathrm{mg} / \mathrm{kg}$ aliment tout au long de la croissance selon les recommandations de l'INRA (1989). Les résultats de Paboeuf et

Figure 20. Spéciation du zinc et du cuivre dans 9 effluents porcins (d'après Morvan et

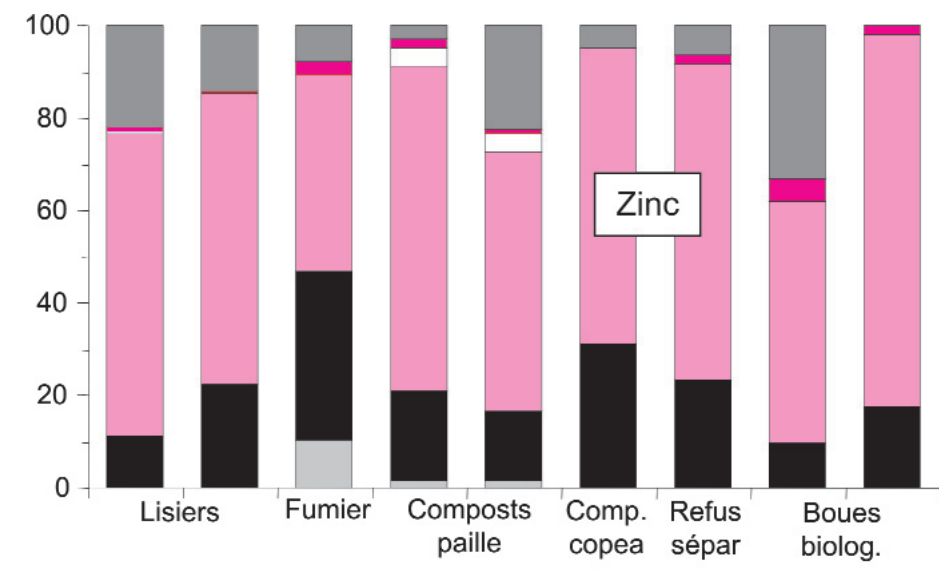

\begin{tabular}{|lll|}
\hline$\square$ Soluble & $\square$ Echangeable & Réductible \\
$\square$ Oxydable & Résiduelle & Colloïdale \\
\hline
\end{tabular}

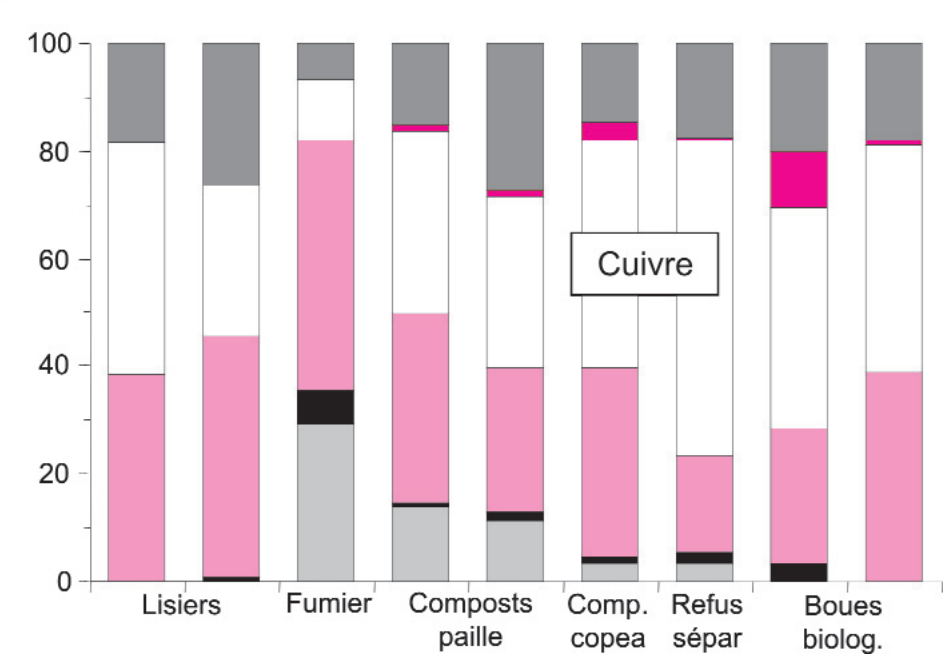


Figure 21. Cinétique de mobilisation $d u$ cuivre après apport d'effluent au sol (d'après Jaffrézic et al 2005).

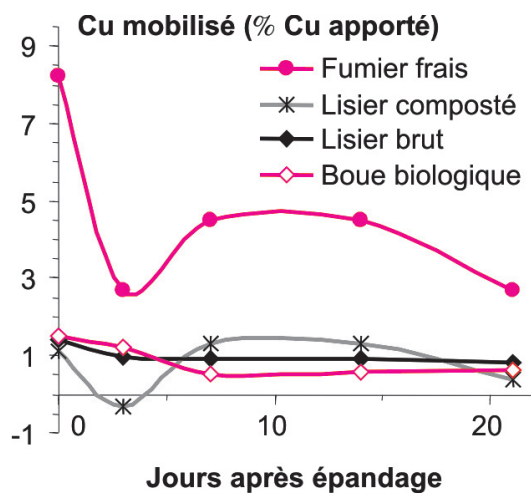

Jondreville (2005) et de Révy et al (2004a et b, 2006) montrent que de tels apports, qui correspondent au contenu des matières premières alimentaires et ne demandent donc pas de supplémentation en cuivre, sont compatibles avec des performances de croissances non altérées.

L'application stricte de la réglementation actuelle de l'UE conduit à épandre sur le sol 12 fois plus de cuivre que les cultures ne peuvent en exporter si le lisier est brut et 24 fois plus si le lisier est traité (figure 22b). Des apports réduits à $10 \mathrm{mg} / \mathrm{kg}$ tout au long de la croissance (Besoin), conduisent encore à appliquer 3 fois plus de cuivre que n'en exportent les cultures si le lisier est épandu brut. Cet excès est multiplié dès qu'un traitement est mis en œuvre pour abattre une partie de l'azote sans capter les métaux dans des coproduits exportable. Dans le cas le plus défavorable, les concentrations de $\mathrm{Cu}$ dans le sol peuvent atteindre le seuil de phytotoxicité en moins de 25 ans (figure 22c). Dans le cas le plus favorable (lisier brut d'animaux nourris selon la stratégie «Besoin»), il faut près d'un millénaire pour atteindre ce seuil.

Pour le zinc, les auteurs ont comparés les stratégies suivantes (figure 22a) :

- UE anc+ : apports selon l'ancienne réglementation de l'UE, sauf pendant la période immédiatement après le sevrage où l'on couvre le risque pathologique avec des teneurs élevées en zinc (cf. §3.1.a) ;

- UE anc : apports selon l'ancienne réglementation de l'UE, en vigueur jusqu'en 2003 ;

- UE : apports selon la réglementation actuelle de l'UE (EC, 1334/2003) ;

- Besoin-Phytase : apports selon les besoins des animaux diminuées de
Figure 22. Relations entre les apports d'éléments traces métalliques sur le sol et les capacités d'exportation par les cultures en fonction des stratégies d'apport alimentaire de zinc et de cuivre (d'après Dourmad et Jondreville 2007).

Figure 22a. Teneurs des aliments en zinc et en cuivre selon du poids vif des porcs.
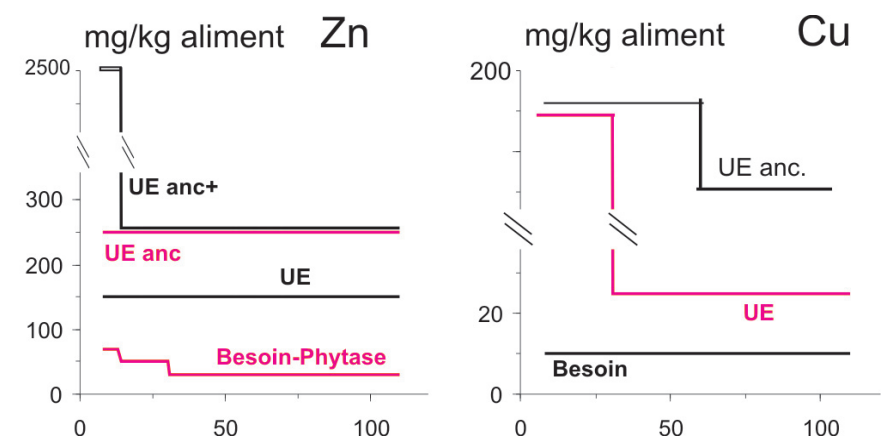

FIgure 22b. Comparaison des quantités de zinc et de cuivre apportées sur le sol avec les capacités d'exportation par les cultures dans le cas d'un lisier brut ( $\mathbf{0}$ ) ou d'un lisier ayant subit un traitement sans séparation de phases abattant $50 \%$ de l'azote ( $\square$ ). Dans les deux cas les quantités d'effluent épandues apportent $170 \mathrm{~kg}$ azote à l'ha.

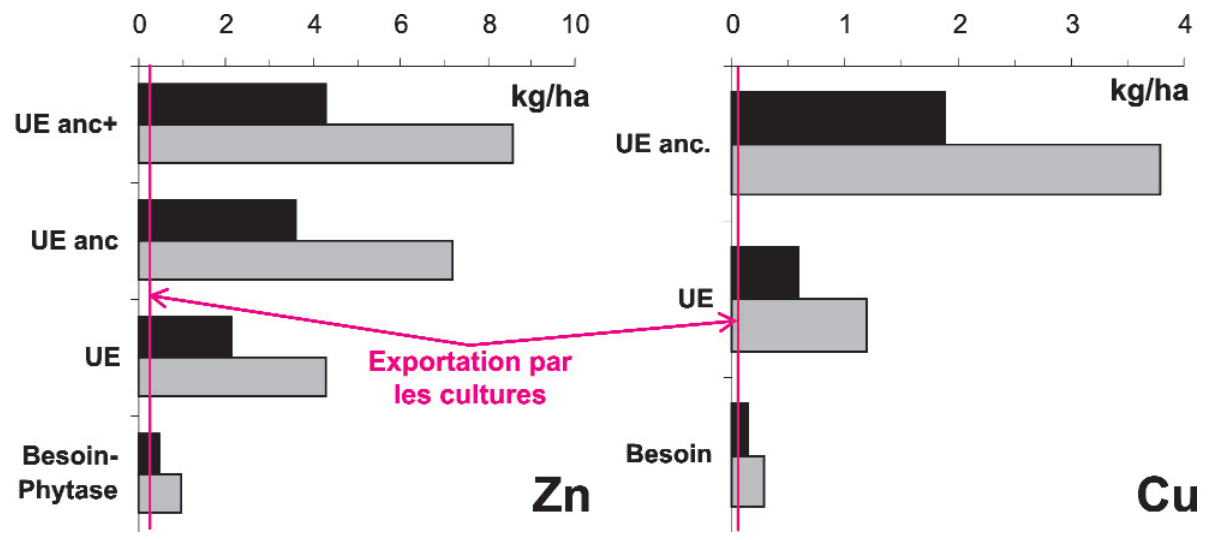

Figure 22c. Nombre d'années d'application de lisier brut ( $\mathbf{\square}$ ) ou de lisier ayant subit un traitement sans séparation de phases abattant 50\% de l'azote ( $\square$ ) au bout desquelles les teneurs dans le sol atteindront $150 \mathrm{mg}$ de $\mathrm{Zn}$ ou $50 \mathrm{mg}$ de Cu par $\mathrm{kg} \mathrm{MS}$. Dans les deux cas les quantités d'effluent épandues apportent $170 \mathrm{~kg}$ azote à l'ha.
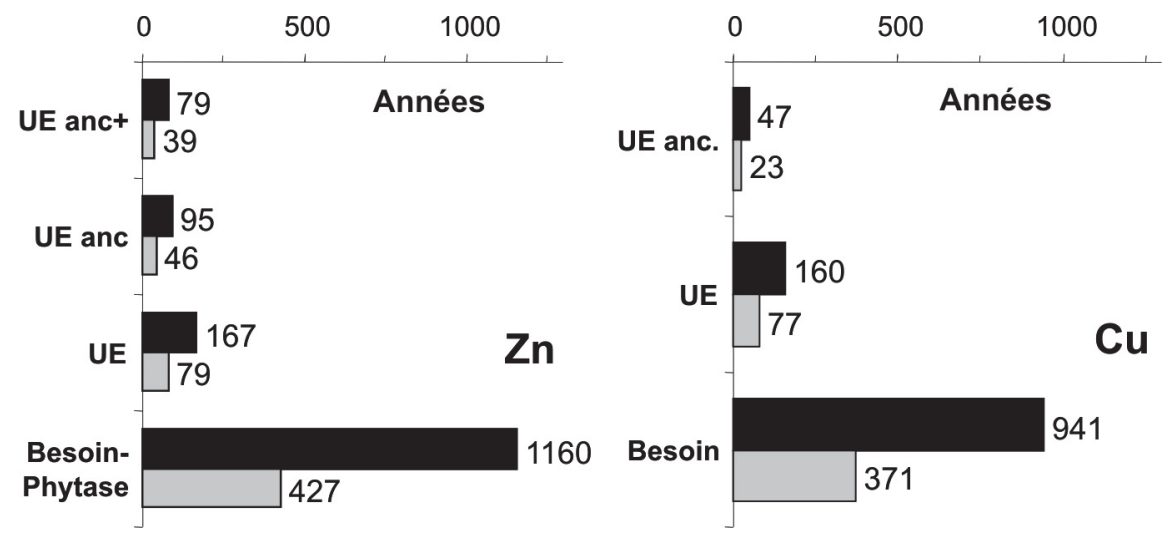

l'épargne autorisée par l'adjonction de phytase microbienne (cf. §3.2).

Même dans le cas le plus favorable (Besoin-Phytase), les quantités de zinc apportées au sol dépassent nettement les capacités d'exportation par les cul- tures (figure 22c). L'épandage de lisier sur la base de $170 \mathrm{~kg}$ d'azote par ha aboutit ainsi dans tous les cas à une accumulation irréversible de zinc dans les sols. Si le lisier a préalablement subi un traitement qui abat une partie importante de l'azote sans capter les métaux 
dans des coproduits exportables, les quantités de zinc épandues excèdent de façon encore plus dramatique les capacités d'exportation des cultures. Dans le cas le plus défavorable, les concentrations de $\mathrm{Zn}$ dans le sol peuvent atteindre le seuil de phytotoxicité en moins d'un demi-siècle d'apport continu d'effluents (figure 22c). Dans le cas le plus favorable (lisier brut d'animaux nourris selon la stratégie «BesoinPhytase»), il faut plus d'un millénaire pour atteindre ce seuil.

\section{4 / Synthèse des connaissan- ces acquises sur les rejets d'azote, phosphore et élé- ments traces métalliques tout au long de la filière de production}

Les connaissances apportées par le programme «Porcherie verte» concernant les cycles des éléments à risques pour l'environnement sont résumées aux figures 23 (azote), 26 (phosphore) et 27 (zinc et cuivre).

L'alimentation est l'un des facteurs majeurs de contrôle des quantités d'éléments excrétés par les animaux. La réduction de $16 \%$ à $12 \%$ de la teneur en protéines de l'aliment permettrait de réduire substantiellement les rejets d'azote dans l'environnement. Cependant, une réduction aussi importante est peu concevable dans le contexte économique actuel compte tenu du prix des acides aminés industriels qu'il faut ajouter à l'aliment à faible teneur en protéines. Une réduction moins poussée (13-14\% de protéines), en particulier pour les aliments de finition, parait plus facilement envisageable dans le contexte économique actuel. Il est, par contre économiquement aisé d'optimiser les teneurs en phosphore dans les aliments et même d'épargner du phosphore par la supplémentation en phytase microbienne. Dans le nouveau contexte d'interdiction des supplémentations en antibiotiques dans les aliments, la réduction des teneurs en zinc et en cuivre à des niveaux compatibles avec les capacités d'exportation par les cultures est un véritable défi. Les apports alimentaires de zinc, mêmes réduits au maximum (scenario NRC-Phytase au §3.5) aboutissent à des quantités épandues qui dépassent les capacités d'exportation par les cultures si les effluents sont épandus sur la base de $170 \mathrm{~kg}$ d'azote à l'hectare. Concernant le cuivre, seuls des ali- ments sans cuivre ajouté (environ 5-6 $\mathrm{ppm}$ de cuivre/kg d'aliment), qui ne sont pas forcément suffisamment sécurisant en matière de croissance du porcelet, aboutissent à des quantités épandues qui ne dépassent pas trop les capacités d'exportation par les cultures. Le traitement des effluents par des procédés n'impliquant aucune séparation de phases (compostage, traitement biologique simple...) ne fait qu'aggraver la situation. Seuls des procédés de séparation de phases sophistiqués (stations de type 3 ou 4 au §3.3) permettent de capter les éléments traces métalliques dans des coproduits potentiellement exportables. Mais les concentrations des coproduits en éléments traces métalliques approchent alors dangereusement ou même dépassent les limites règlementaires, ce qui peut limiter en pratique les possibilités de valorisation de ces coproduits. Compte tenu de ces difficultés de maîtrise des éléments traces métalliques, il apparaît impératif de rechercher des méthodes d'élevage plus favorables au maintien de la santé des animaux (alimentation, conditions d'ambiance), en particulier des porcelets pendant la période de post-sevrage.

L'utilisation de litières et le compostage entraînent des pertes d'azote très importantes. Pour les éleveurs qui ont des excédents d'azote organique dans les effluents par rapport aux surfaces épandables dont ils disposent, les litières et le compostage sont des moyens très efficaces pour abattre l'azote, qui peuvent être bien adaptées à des exploitations moyennes ne pouvant pas investir dans une station de traitement. Ces solutions présentent des avantages connexes (réduction des nuisances olfactives, amélioration de l'image et du bien-être des animaux) mais aussi de sérieux inconvénients :

- litières et compostage sont source d'émissions de gaz à effet de serre (cf. article sur les émissions gazeuses) à des niveaux qui peuvent devenir élevés si l'on cherche à faire trop d'économies sur la quantité de substrat carboné (augmentation de la densité animale, litières en couche mince, apports insuffisant de substrat additionnel sur des litières en couche épaisses, compostage en couche fine) ;

- l'abattement d'azote correspond à une perte d'éléments fertilisants de grande valeur. Pour les éleveurs qui ne sont pas en excédent, il y a là une perte économique substantielle car il faudra compenser par l'achat d'engrais minéraux. A un niveau plus global, le «gaspillage» d'éléments fertilisants ainsi réalisé correspond à un impact défavorable sur l'environnement. En effet, l'azote perdu aurait permis d'épargner des engrais azotés minéraux qui coûtent cher en énergie aussi bien pour leur production que pour leur transport. Or, la production d'énergie est très impactante sur l'environnement (cf. conclusion de l'article sur les émissions gazeuses) ;

- litières et compostage sont sans effet sur les éléments traces métalliques (cf. supra) et sur le phosphore. Ils ne constituent donc pas une solution viable pour résoudre les excédents structurels dès lors que la réglementation n'est pas limitée à l'azote. La dégradation des substrats carbonés dans la litière et lors du compostage et l'abattement d'azote concomitant aboutissent à une concentration du phosphore, du cuivre et du zinc dans le produit à épandre. Par exemple, s'agissant du compostage de lisier sur paille (méthode "Guenévez»), les concentrations dans le produit frais obtenues à la fin de la phase d'imprégnation sont multipliées par $1,5,1,3,1,5,1,4$ pour $\mathrm{P}, \mathrm{K}, \mathrm{Cu}$ et $\mathrm{Zn}$, respectivement, ces produits n'étant pas volatils. Ces concentrations font de ce produit composté un engrais organique très riche dont il faut raisonner les apports, notamment en $\mathrm{N}$ et P. Le produit restant humide (25\% de MS), il peut être délicat à appliquer avec la précision des épandeurs actuels et difficile à exporter de l'exploitation. Après la maturation, les concentrations sont encore accrues dans le compost; elles sont multipliées par 2,4, 1,7, 2,5 et 1,9 pour $\mathrm{P}, \mathrm{K}, \mathrm{Cu}$ et $\mathrm{Zn}$, respectivement. Cependant, le compost est plus sec (35\% de MS) et sera donc plus facile à épandre.

La capacité des procédés de traitement biologique à abattre l'azote en font des instruments intéressants de gestion des effluents aux échelles locales, dans les situations d'excès par rapport aux capacités d'épandage (par exemple dans les Zones d'Excédents Structurels (ZES)). Le raisonnement des autorisations d'épandage sur la seule base de l'azote organique peut cependant conduire à des situations catastrophiques en termes de quantités de phosphore ou d'éléments traces métalliques apportés au sol si aucune séparation de phases n'est opérée. Mais, même dans ce cas, les coproduits ainsi obtenus peuvent être de facto difficilement exportables compte tenu de leurs teneurs en éléments traces métalliques (cf. supra). Enfin la destruction, à un coût énergétique élevé, d'éléments fertilisants azotés dont la fabrication 
Figure 23. Connaissances acquises dans le cadre du programme "Porcherie verte» sur le devenir de l'azote dans une exploitation porcine (commentaires en rouge). Les commentaires en noir correspondent à des connaissances acquises antérieurement.

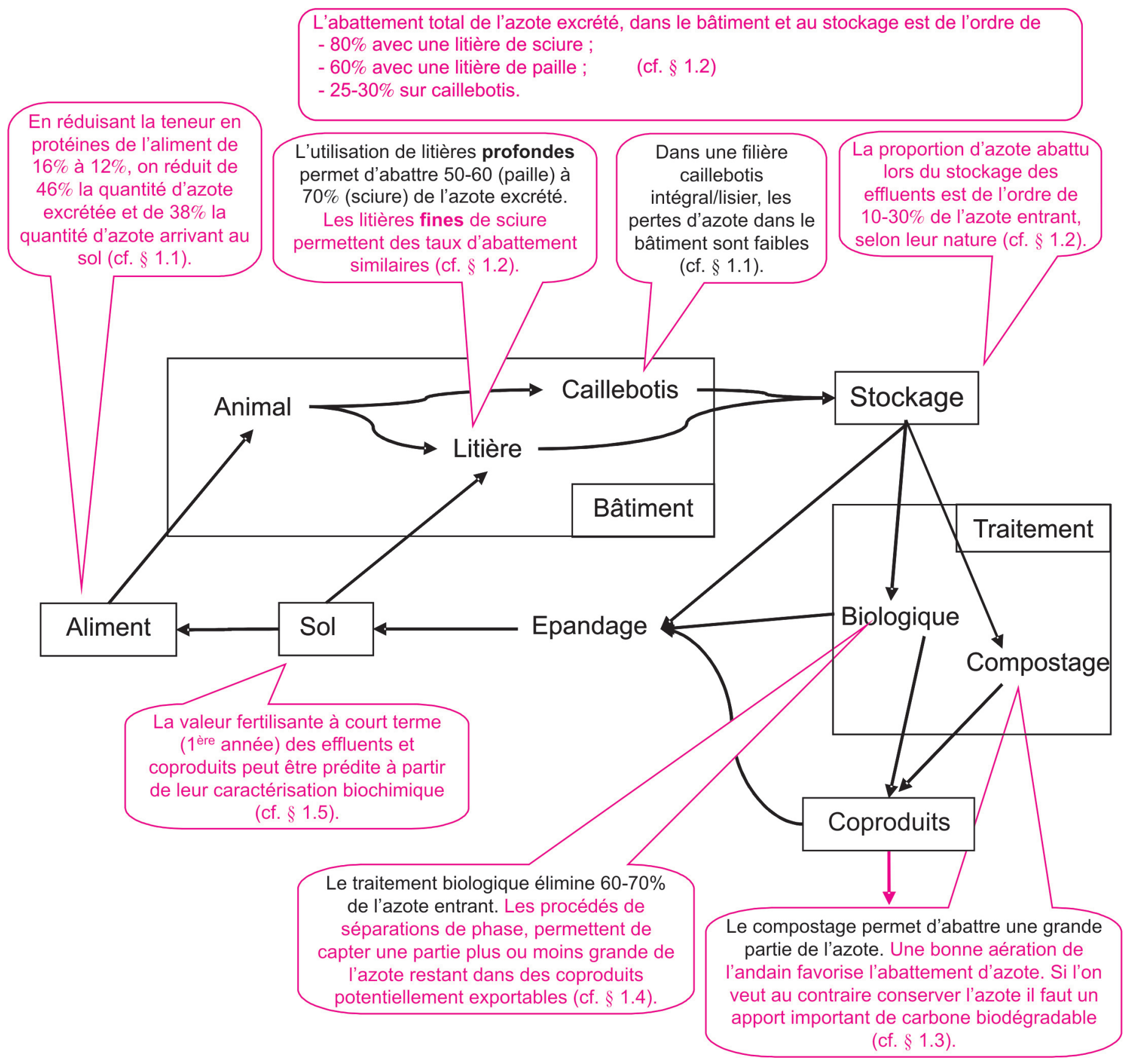

sous forme d'engrais minéraux est ellemême très exigeante en énergie, constitue, à grande échelle, un paradoxe écologique et économique qui pourrait être difficile à soutenir à long terme.

Ainsi, traitement biologique, litières et compostage souffrent en gros des mêmes inconvénients en termes d'impact écologique et de coût avec cependant des nuances importantes :

- le traitement a un coût économique plus élevé, surtout pour les petites exploitations qui doivent amortir des investissements très élevés sur des volumes traités plus faibles ;

- le traitement conduit à des émissions de gaz à effet de serre plus faibles sur l'exploitation (cf. article sur les émissions gazeuses), mais l'énergie nécessaire au traitement est elle-même très impactante sur l'environnement ;

- sous réserve d'être associé à une séparation de phases, le traitement peut abattre aussi le phosphore et même des éléments traces métalliques si des techniques de séparation très poussées sont mises en oeuvre.

\section{Conclusions}

En fin de compte, la manière la plus simple et la plus économique de gérer les effluents d'élevage reste d'utiliser au mieux leur valeur fertilisante. En ce sens la filière «Lisier» ne manque pas d'atouts car c'est elle qui préserve le mieux les quantités d'azote et de phosphore rejetés par les animaux. Ceci sous réserve d'un bon équilibre entre le volume de production porcine et les surfaces épandables portant des cultu- 
Figure 24. Connaissances acquises dans le cadre du programme "Porcherie verte» sur le devenir du phosphore dans une exploitation porcine (commentaires en rouge). Les commentaires en noir correspondent à des connaissances acquises antérieurement.

La teneur optimale en phosphore digestible peut être déduite des besoins de l'animal (cf. $\S 2.1$ ).

La connaissance précise de la digestibilité du phosphore dans les matières premières alimentaires permet d'ajuster les apports (cf. §2.2.a) et de raisonner la pertinence d'une supplémentation en phytase microbienne qui améliore la digestibilité (cf. § 2.2.b).

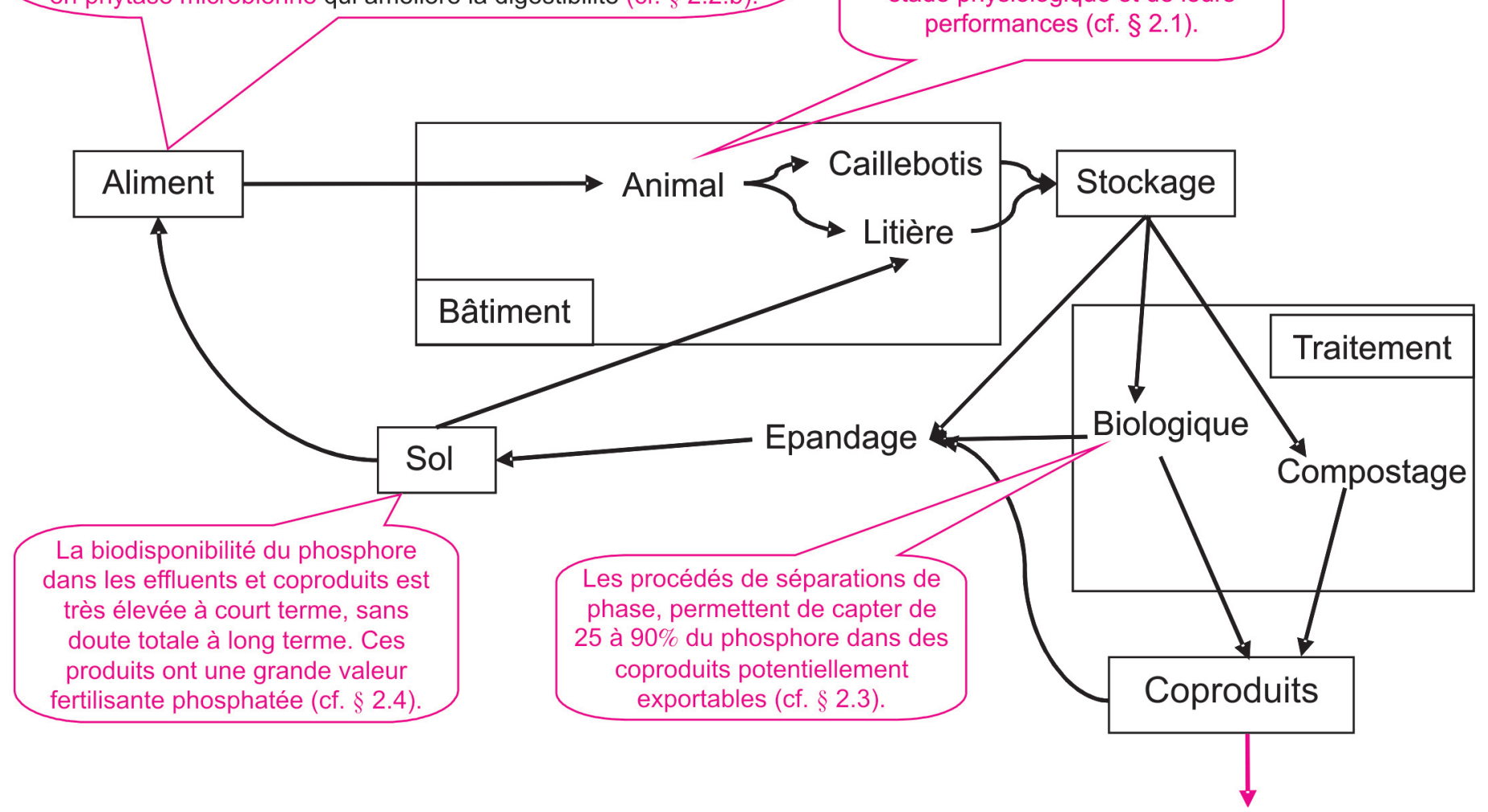

res susceptibles de recevoir ce lisier (cf. $\$ 2$ de l'article sur l'évaluation globale des systèmes de production). Lorsque les quantités d'éléments à épandre excèdent la capacité d'accueil des surfaces épandables, la mise en œuvre de traitements représente une charge économique importante et constitue un non-sens écologique (cf. supra). Le lisier se gère comme un engrais minéral car les formes d'azote qu'il contient sont très majoritairement immédiatement disponibles pour les plantes. Il faut donc bien gérer le calendrier des apports sur les surfaces en fonction des besoins des cultures. La filière «Lisier» souffre cependant d'un certain nombre d'inconvénients d'ordre agronomique (pauvre en carbone et en azote organisé, il participe peu à améliorer la structure des sols) et surtout zootechnique. L'élevage sur caillebotis est réputé défavorable au bien-être animal et les lisiers sont sources de nuisances olfactives importantes (cf. article sur les nuisances olfactives).

Les filières «Litières» permettent de répondre à ces préoccupations. Mais elles présentent cependant l'inconvénient de conduire à des pertes d'azote importantes qui grèvent indirectement le budget des exploitations qui ne sont pas en excédent structurel au travers des achats d'engrais minéral qu'il faut consentir pour les compenser. Ces pertes d'azote sont couramment mises à profit par certains éleveurs pour abattre les quantités d'azote excédentaires par rapport à la surface épandable dont ils disposent. En ce sens la litière (ou le compostage) peut à première vue constituer une alternative séduisante pour ceux qui ne veulent pas, ou ne peuvent pas, s'engager dans les investissements importants que représente une station de traitement biologique. Tout comme le traitement biologique simple (sans séparation de phases), la litière et le compostage, vus sous le seul angle de technique permettant d'abattre l'azote, ne sont pas des solutions durables car ils ne permettent pas d'abattre le phosphore et ne répondent donc pas aux exigences des réglementations qui prennent en compte cet élément en plus de l'azote. Il faut donc chercher ailleurs (nuisances olfactives, bien-être animal, image) les véritables raisons d'adopter la litière. La valorisation des fumiers et des composts en tant que fertilisants se gèrent de façon très différente du lisier. Ils participent de façon significative à l'amélioration de la structure des sols, mais les formes d'azote qu'ils contiennent sont majoritairement non immédiatement disponibles pour les plantes et il faut prendre en compte leur dynamique de minéralisation. 
Figure 25. Connaissances acquises dans le cadre du programme «Porcherie verte» sur le devenir du zinc et du cuivre dans une exploitation porcine (commentaires en rouge). Les commentaires en noir correspondent à des connaissances acquises antérieurement.

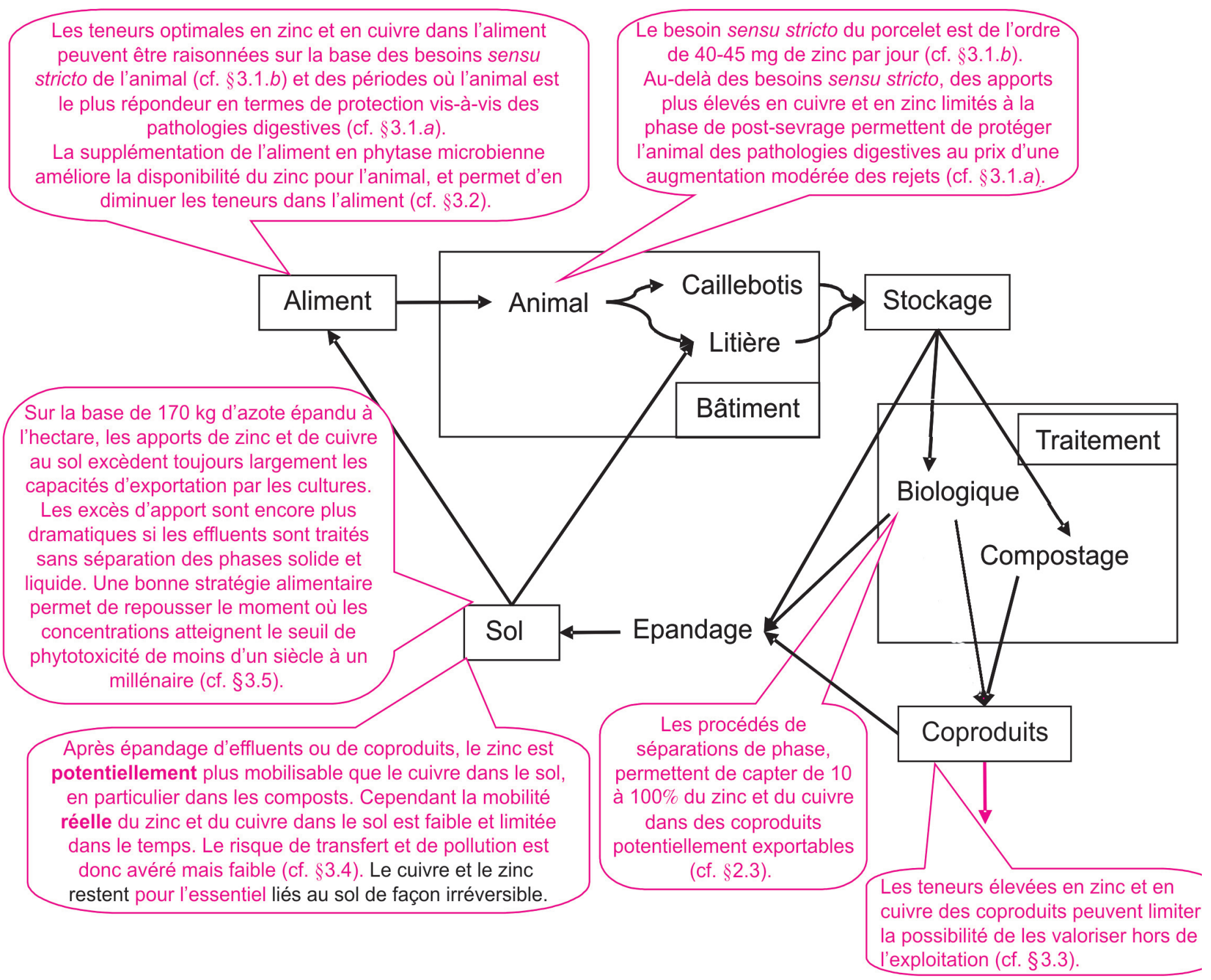

\section{Références}

Abd El Kader N., Robin P., Paillat J.M., Leterme P., 2007. Turning, compacting and the addition of water as factors affecting gaseous emissions in farm manure composting. Bioresource Technol., 98, 2619-2628.

Béline F., Daumer M.L., Guiziou F., 2003. Performances des principales filières de traitement biologique aérobie du lisier de porcs. Journ. Rech. Porcine Fr., 35, 29-34.

Braude R., 1975. Copper as a performance promoters in pig. Copper in farming. Symposium, Royal Zool. Soc., 24 sept., 79-97.

Cambeilh D., Meymerit C., Cazaux J.G., Castaing J., Skiba F., 2005. Incidence de la réduction de l'apport de phosphore dans les aliments pour les truies en gestation et en lactation. Journ. Rech. Porcine Fr., 37, 7-16.

Castaing J., Paboeuf F., Skiba F., Chauvel J., Cazaux J.G., van Milgen J., Jondreville C., 2003.
Estimation du besoin en phosphore digestible apparent du porc charcutier : synthèse d'essais zootechniques effectués au cours des dix dernières années. Journ. Rech. Porcine Fr., 35, 47-54.

Coppenet M., Golven J., Simon J.C., Le Roy M., 1993. Evolution chimique des sols en exploitations d'élevage intensif : exemple du Finistère. Agronomie, 13, 77-83.

CORPEN, 2003. Estimation des rejets d'azote - phosphore - potassium - cuivre et zinc des porcs. MAAPAR - MEDD, 44p.

Dourmad, J.Y., Jondreville, C., 2007. Impact of nutrition on nitrogen, phosphorus, $\mathrm{Cu}$ and $\mathrm{Zn}$ in pig manure, and on emissions of ammonia and odours. Livest. Sci., 112, 192-198.

EC 1334/2003. Commission Regulation EC $N^{\circ} 1334 / 2003$ amending the conditions for authorization of a number of additives in feedingstuffs belonging to the group of trace elements. Off. J. Eur. Union L., 187, 11-15.

EDE Bretagne, 1998. Compostage selon la méthode «Guernévez». Mesure des pertes d'ammoniac pendant la phase de compostage actif. Chambres d'Agriculture de Bretagne, 5p.

Espagnol S., Hassouna M., Robin P., Levasseur P., Paillat J.M., 2006. Emissions gazeuses de $\mathrm{NH}_{3}, \mathrm{~N}_{2} \mathrm{O}, \mathrm{CH}_{4}$ lors du stockage de fumier de porc provenant d'une litière accumulée : effets du retournement. Journ. Rech. Porcine Fr., 38, 41-48.

Guéguen L., Perez J.M., 1981. A re-evaluation of recommended dietary allowances of calcium and phosphorus for pigs. Proc. Nutr. Soc., 40 273-278.

INRA, 1989. Alimentation des porcs en croissance. In : L'alimentation des animaux monogas- 
triques : Porc, Lapin, Volailles. Inra Editions, Paris, France, 49-66.

Jaffrézic A., Cornu S., Racapé A., Morvan T., 2005. Trace elements speciation in farming organic wastes and risk of mobility in soils. Int Workshop on green pork production, May 25-27, Paris, France, 83-84.

Jondreville C., 2003. Réduction par l'alimentation des rejets de phosphore et d'éléments traces métalliques (cuivre et zinc). Rapport de synthèse de l'action $\mathrm{AC} 32 \mathrm{e} \mathrm{du}$ programme «Porcherie verte», 20p.

Jondreville C., Dourmad J.Y., 2005. Le phosphore dans la nutrition des porcs. INRA Prod. Anim., 18, 183-192.

Jondreville C., Revy P.S., Dourmad J.Y., 2003. Dietary means to better control the environmental impact of copper and zinc by pigs from weaning to slaughter. Livest. Prod. Sci., 84, 147-156.

Jondreville C., Revy P.S., Dourmad J.Y., Nys Y., Hillion S., Pontrucher F., Gonzalez J., Soler J., Lizardo R., Tibau J., 2004. Influence du sexe et du génotype sur la rétention corporelle de calcium, phosphore, potassium, sodium, magnésium, fer, zinc et cuivre chez le porc de 25 à $135 \mathrm{~kg}$ de poids vif. Journ. Rech. Porcine Fr., 36, 17-24.

Jondreville C., Skiba F., Castaing J., Chauvel J., Paboeuf F., Dourmad J.Y., 2005a. Digestible P requirement by growing-finishing pigs. Int. Workshop on green pork production, May 25-27, Paris, France, 71-72.

Jondreville C., Hayler R., Feuerstein D., 2005b. Remplacement du sulfate de zinc par de la phytase microbienne dans des aliments pour porcelets sevrés. Journ. Rech. Porcine Fr., 37, $17-$ 24.

Jondreville C., Hayler R., Feuerstein D., 2005 c. Replacement of zinc sulphate by microbial phytase for piglets given a maize-soya-bean based diet. Anim. Sci., 81, 77-83.

Linères M., Manga A., Morel C., 2005. Plant availability of phosphorus from pig wastes. Int. Workshop on green pork production, May 25-27, Paris, France, 75-76.
Morvan T., Nicolardot B., 2005. Biochemical composition and kinetics of $\mathrm{C}$ and $\mathrm{N}$ mineralization of animal wastes: a typological and modelling approach. Int. Workshop on green pork production, May 25-27, Paris, France, 67-68.

Morvan T., Jaffrezic A., Linères M., 2004 Quantification des flux d'éléments à risques : Azote, Phosphore et Eléments Traces Métalliques. Rapport de synthèse de l'action AC43a du programme «Porcherie verte», 69p.

Morvan T., Nicolardot B., Péan L., 2006. Biochemical composition and kinetics of $\mathrm{C}$ and $\mathrm{N}$ mineralization of animal wastes: a typological approach. Biol. Fertil. Soils, 42, 513-522.

Paboeuf F., Jondreville C., 2005. Effect of the level of copper and zinc supplied to piglets and growing-finishing pigs on their performance and excretion in manure. Int. Workshop on green pork production, May 25-27, Paris, France, 8182 .

Paillat J.M., Robin P., Hassouna M., Leterme P., 2005a. Effet du compostage d'effluents porcins sur les émissions gazeuses et les teneurs en éléments polluants. Rapport de synthèse de l'action $\mathrm{AC} 42 \mathrm{c}$ du programme «Porcherie verte», $106 \mathrm{p}$

Paillat J.M., Robin P., Hassouna M., Leterme P., 2005b. Predicting ammonia and carbon dioxide emissions from carbon and nitrogen biodegradability during animal waste composting. Atmosph. Env., 39, 6833-6842.

Portejoie S., Dourmad J.Y., Martinez J. Lebreton Y., 2002. Effet de la réduction du taux protéique de l'aliment sur la volatilisation ammoniacale des effluents porcins. Journ. Rech. Porcine Fr., 34, 167-174.

Portejoie S., Dourmad J., Martinez J., Lebreton Y., 2004. Effect of lowering dietary crude protein on nitrogen excretion, effluent composition and ammonia emission from fattening pigs. Livest. Prod. Sci., 91, 45-55.

Ramonet Y., Callarec J., 2005. Perte d'azote du fumier au cours de la période d'engraissement de porcs sur litière puis lors de la phase de stockage. Journ. Rech. Porcine Fr., 37, 33-38.
Ramonet Y., Robin P., 2002. L'engraissement de porcs sur litière de particules de bois ou de sciure en couche fine. Journ. Rech. Porcine Fr., $34,143-148$

Revy P.S., Jondreville C., Dourmad J.Y. Guinotte F., Coupel A., Nys Y., 2003. Influence des phytates sur la disponibilité du zinc issu de différentes sources chez le porcelet sevré. Journ. Rech. Porcine Fr., 35, 55-60.

Revy P.S., Jondreville C., Dourmad J.Y., Nys Y., 2004a. Apports alimentaires de zinc nécessaires à la couverture du besoin du porcelet sevré recevant un aliment à base de maïs et de tourteau de soja additionné ou non de phytase microbienne. Journ. Rech. Porcine Fr., 36, 25-32.

Revy P.S., Jondreville C., Dourmad J.Y., Nys Y., 2004b. Effect of zinc supplemented as either an organic or an inorganic source and of microbial phytase on zinc and other minerals utilisation by weanling pigs. Anim. Feed Sci. Technol., 116 93-112.

Revy P.S., Jondreville C., Dourmad J.Y., Nys Y., 2006. Assessment of dietary zinc requirement of weaned piglets fed diets with or without microbial phytase. J. Anim. Physiol. Anim. Nutr., 90, 50-59.

Robin P., Hassouna M., Ramonet Y., Texier C. 2004b. Maîtrise des émissions gazeuses en bâtiments sur litière (validation en élevages des résultats acquis en conditions climatiques contrôlées). Rapport de synthèse de l'action AC41b du programme «Porcherie verte», $110 \mathrm{p}$.

Sauvant D., Perez J.M., Tran G., 2002. Tables de composition et de valeur nutritive des matières premières destinées aux animaux d'élevage porcs, volailles, bovins, ovins, caprins, lapins, chevaux, poissons. INRA Editions, Paris, 301p.

Skiba F., Callu P., Castaing J., Paboeuf F. Chauvel J., Jondreville C., 2004. Variabilité intra-matière première de la digestibilité du phosphore des céréales et du pois chez le porc en croissance. Journ. Rech. Porcine Fr., 36, 9-16.

Van-Soest P.J., 1963. Use of detergents in the analysis of fibrous feeds. II. A rapid method for the determination of fibre and lignin. J. Ass. Anal. Chemist., 46, 829-835.

\section{Résumé}

Cet article résume les apports du programme «Porcherie verte» dans la connaissance et la maîtrise du devenir de l'azote, du phosphore et des éléments traces métalliques qui ont, à des titres divers, un impact sur l'environnement.

La diminution de la teneur en protéines de l'aliment permet de réduire fortement les quantités d'azote excrétées par les animaux. Une part importante de l'azote est éliminée sous forme gazeuse lorsque les animaux sont placés sur litière ou lorsque le lisier est composté. L'importance de ces pertes d'azote peut cependant varier fortement selon les techniques utilisées et la nature des substrats. Le traitement biologique abat la majeure partie de l'azote et certains types de stations permettent de capter le reste dans des coproduits potentiellement exportables. La bonne valorisation agronomique des effluents nécessite de bien connaître leur valeur fertilisante azotée, ce que facilite l'approche typologique mise au point dans le cadre du programme.

Un certain nombre de leviers alimentaires permettent de diminuer la fraction du phosphore alimentaire qui est excrétée dans les effluents : ajustement des apports alimentaires grâce à une meilleure connaissance des besoins des animaux, amélioration de la digestibilité du phosphore alimentaire par une meilleure connaissance de sa disponibilité dans les diverses matières premières ou via l'adjonction de phytase exogène. Les traitements biologiques avec séparation de phases permettent de capter le phosphore dans des coproduits potentiellement exportables et la valeur fertilisante phosphatée des effluents est en général très élevée et facile à prédire.

Les éléments traces métalliques (cuivre et zinc) sont souvent ajoutés dans l'aliment à des concentrations dépassant largement les besoins stricts des animaux (pour éviter les carences) afin de bénéficier de leur effet protecteur vis-à-vis des pathologies digestives. La supplémentation par des éléments traces métalliques est utile pendant la phase de post-sevrage, mais pas au-delà et l'adjonction de phytase microbienne à l'aliment améliore la disponibilité du zinc pour l'animal. Les traitements biologiques avec séparation de phases permettent de capter le zinc et le cuivre dans des coproduits potentiellement exportables. Après épandage, les éléments traces métalliques sont peu mobiles dans le sol alors que les apports au sol excèdent en général largement les capacités d'exportation par les plantes, ce qui peut conduire à des situations de phytotoxicité à plus ou moins long terme. 
En fin de compte, la manière la plus simple et la plus économique de gérer les effluents d'élevage reste d'utiliser au mieux leur valeur fertilisante, ce qui s'obtient par un bon équilibre entre la quantité d'animaux produits et la capacité des sols à recevoir leurs effluents. En l'absence d'un tel équilibre, l'abattement de l'azote excédentaire par l'utilisation de litières ou par le compostage du lisier a un impact environnemental important alors même que ces solutions ne résolvent rien en termes de phosphore et d'éléments traces métalliques. Les traitements biologiques les plus sophistiqués permettent d'éliminer le phosphore et une partie des éléments traces métalliques dans des coproduits potentiellement exportables, mais ils ont un coût économique et écologique très élevé.

\section{Abstract \\ Elements of waste from pigs : knowledge and control}

This paper summarises the contributions that the «Porcherie verte» programme makes to our knowledge and control of nitrogen, phosphorus and trace metals, which each have an impact on the environment.

The decrease in protein content of feed reduces the amount of nitrogen excreted by animals. An important part of nitrogen is eliminated as a gas when the animals are placed on floor litter or when the manure is used as compost. The importance of these nitrogen losses, however, varies according to the techniques used and the nature of the substrates. The biological treatment capts most of the nitrogen and some farms can capt the rest in potentially exportable co-products. The good agronomic valorisation of waste requires good knowledge of the nitrogen fertilisation value, which makes the typological approach developed within the framework of the programme easier.

A certain number of food levers decrease the fraction of food phosphorus that is excreted in waste: adjusting food intake by a better knowledge of the animal's needs, improvement of digestibility of food phosphorus by improved knowledge of its availability in different raw materials or by adding exogenous phytase. Biological treatments with phase separation capt phosphorus in potentially exportable co-products and phosphate fertilising value of waste is generally very high and easy to predict.

Trace metals (copper and zinc) are often added to feed in concentrations much higher than those needed by the animal (to avoid deficiency) in order to benefit from their protective effects against digestive pathologies. Supplementation of trace metals is useful during the postweaning phase, but not beyond and the addition of microbial phytase in feed improves the availability of zinc for the animal. Biological treatments with phase separation capture zinc and copper in the potentially exportable co-products. After spreading, trace metals are little mobile in the soil whereas soil supply generally exceeds the export capacities of plants. This can therefore lead to phytotoxicity situations on a short to long term.

Finally, the best way and most economic to manage breeding waste is the adapted use of fertilising value, which is obtained by a good balance between the quantity of animal products and the capacity of soil to absorb waste. Without such a balance, capting excess waste by using litter or composting manure has an important impact on the environment even though these solutions do not resolve anything in terms of phosphorus and trace metals. The most sophisticated biological treatments eliminate phosphorus and a part of the trace metals in the potentially exportable co-products but they have a very high cost for the economy and ecology.

BONNEAU M., BELINE F., DOURMAD J.-Y., HASSOUNA M., JONDREVILLE C., LOYON L., MORVAN T., PAILLAT J.-M., RAMONET Y., ROBIN P., 2008. Connaissance du devenir des éléments à risques dans les différentes filières de gestion des effluents porcins. INRA Prod. Anim., 21, 325-344. 
